\title{
Modeling Sampling in Tensor Products of Unitary Invariant Subspaces
}

\author{
Antonio G. García, ${ }^{1}$ Alberto Ibort, ${ }^{1}$ and María J. Muñoz-Bouzoº \\ ${ }^{1}$ Departamento de Matemáticas, Universidad Carlos III de Madrid, Avda. de la Universidad 30, Leganés, 28911 Madrid, Spain \\ ${ }^{2}$ Departamento de Matemáticas Fundamentales, U.N.E.D., Senda del Rey 9, 28040 Madrid, Spain \\ Correspondence should be addressed to Antonio G. García; agarcia@math.uc3m.es
}

Received 25 July 2016; Accepted 16 October 2016

Academic Editor: Hans G. Feichtinger

Copyright (C) 2016 Antonio G. García et al. This is an open access article distributed under the Creative Commons Attribution License, which permits unrestricted use, distribution, and reproduction in any medium, provided the original work is properly cited.

\begin{abstract}
The use of unitary invariant subspaces of a Hilbert space $\mathscr{H}$ is nowadays a recognized fact in the treatment of sampling problems. Indeed, shift-invariant subspaces of $L^{2}(\mathbb{R})$ and also periodic extensions of finite signals are remarkable examples where this occurs. As a consequence, the availability of an abstract unitary sampling theory becomes a useful tool to handle these problems. In this paper we derive a sampling theory for tensor products of unitary invariant subspaces. This allows merging the cases of finitely/infinitely generated unitary invariant subspaces formerly studied in the mathematical literature; it also allows introducing the several variables case. As the involved samples are identified as frame coefficients in suitable tensor product spaces, the relevant mathematical technique is that of frame theory, involving both finite/infinite dimensional cases.
\end{abstract}

\section{Introduction}

Sampling and reconstruction of functions in unitary invariant subspaces of a separable Hilbert space bring a unified approach to sampling problems (see [1-5]). Indeed, it englobes the most usual sampling settings such as sampling in shift-invariant subspaces of $L^{2}(\mathbb{R})$ (see, e.g., [6-17] and references therein) or sampling periodic extensions of finite signals (see $[3,18]$ ).

In a recent paper [19] it was shown how to extend sampling reconstruction theorems to invariant subspaces of separable Hilbert spaces under a unitary representation of finite groups which are semidirect products with an Abelian factor. This setting is appropriate for applications of the theory beyond the domain of classical telecommunications to quantum physics.

In this paper we go one step ahead by enlarging the class of target spaces for sampling: we deal with tensor products of different unitary invariant subspaces. This situation corresponds, for instance, to consider multichannel systems in classical telecommunications or composite systems in the case of quantum applications. Thus, in this setting, we are able to gather problems of diverse nature by means of a simple formalism involving tensor products and tensor operators in Hilbert spaces. Namely, we first consider an infinite $U$-unitary subspace

$$
\mathscr{A}_{a}=\left\{\sum_{n \in \mathbb{Z}} a_{n} U^{n} a:\left\{a_{n}\right\} \in \ell^{2}(\mathbb{Z})\right\}
$$

in a Hilbert space $\mathscr{H}_{1}$ and a finite $V$-unitary subspace

$$
\mathscr{A}_{b}=\left\{\sum_{p=0}^{N-1} b_{p} V^{p} b: b_{p} \in \mathbb{C}\right\}
$$

in a Hilbert space $\mathscr{H}_{2}$, to finally obtain sampling formulas in their tensor product $\mathscr{A}_{a, b}:=\mathscr{A}_{a} \otimes \mathscr{A}_{b}$.

Apart from tensor products in Hilbert spaces, the paper involves the theory of frames. Concretely, in this situation, the generalized samples will be expressed as frame coefficients in an auxiliary Hilbert space $L^{2}(0,1) \otimes \ell_{N}^{2}(\mathbb{Z})$, where $\ell_{N}^{2}(\mathbb{Z})$ denotes the space of all $N$-periodic complex sequences. Continuing the line of inquiry of $[2,3]$, the problem reduces 
to find appropriate families of dual frames. By "appropriate" we mean that these dual frames have a nice structure taking care of the unitary invariance of the involved sampling subspaces as it will be discussed in the sequel.

Later on, the infinite-infinite and finite-finite generator cases will be considered too, that is, the situation where both invariant subspaces are generated by a sequence of vectors $U^{n} a, V^{p} b$, and $n, p \in \mathbb{Z}$ and the simpler case where both subspaces are finite dimensional. Relevant examples of each situation will be discussed in detail.

The paper is organized as follows: for the sake of completeness we include in Section 2 the basics of frames and tensor products needed in the sequel. In Section 3 we focus on the above case that we call infinite-finite generators case; that is, we establish sampling formulas in the tensor product of two unitary invariant subspaces, one of them $\mathscr{A}_{a}$ with an infinite generator $a$ and the other one $\mathscr{A}_{b}$ finitely generated. First, we obtain appropriate expressions for the samples of any $x \in \mathscr{A}_{a, b}$ obtained from $S=s s^{\prime}$ systems $\mathscr{L}_{j j^{\prime}}$ acting on $\mathscr{A}_{a, b}$,

$$
\mathscr{L}_{j j^{\prime}} x(r n, \bar{r} m):=\left\langle x, U^{r n} h_{j 1} \otimes V^{\bar{r} m} h_{j^{\prime} 2}\right\rangle_{\mathscr{H}_{1} \otimes \mathscr{H}_{2}},
$$

where $n \in \mathbb{Z}, m=0,1, \ldots, \ell-1, j=1,2, \ldots, s$, and $j^{\prime}=$ $1,2, \ldots, s^{\prime}$. Here, $h_{j 1}, j=1,2, \ldots, s$, denote $s$ fixed elements in $\mathscr{H}_{1}$ and $h_{j^{\prime} 2}, j^{\prime}=1,2, \ldots, s^{\prime}$, denote $s^{\prime}$ fixed elements in $\mathscr{H}_{2} ; r$ and $\bar{r}$ are the sampling periods, where $r \in \mathbb{N}$ and $\bar{r}$ is a divisor of $N$ and $\ell:=N / \bar{r}$ (see Section 3 for the details). Then we state the suitable isomorphism $\mathscr{T}_{a, b}^{U V}$ between $L^{2}(0,1) \otimes$ $\ell_{N}^{2}(\mathbb{Z})$ and $\mathscr{A}_{a, b}$ which allows transforming the derived frame expansions in $L^{2}(0,1) \otimes \ell_{N}^{2}(\mathbb{Z})$ into stable sampling formulas in $\mathscr{A}_{a, b}$ having the form

$$
\begin{aligned}
& x \\
& =\sum_{j=1}^{s} \sum_{j^{\prime}=1}^{s^{\prime}} \sum_{n \in \mathbb{Z}} \sum_{m=0}^{\ell-1} \mathscr{L}_{j j^{\prime}} x(r n, \bar{r} m)\left(U^{r n} \otimes V^{\bar{r} m}\right)\left(c_{j} \otimes d_{j^{\prime}}\right),
\end{aligned}
$$

where $c_{j} \in \mathscr{A}_{a}, j=1,2, \ldots, s$, and $d_{j^{\prime}} \in \mathscr{A}_{b}, j^{\prime}=1,2, \ldots, s^{\prime}$. We conclude the section giving a representative example arising from classical tomography (see, e.g., [20, 21]).

Sections 4 and 5 deal with the called infinite-infinite and finite-finite cases. They mimic the structure of Section 3 with auxiliary spaces $L^{2}(0,1) \otimes L^{2}(0,1)$ and $\ell_{N}^{2}(\mathbb{Z}) \otimes \ell_{M}^{2}(\mathbb{Z})$, respectively.

Finally, it is worth mentioning that for the sake of simplicity we only deal with the tensor product of two single generated unitary invariant subspaces; the same results apply for the tensor product of any finite number of multiple generated unitary invariant subspaces.

\section{A Brief on Frames and Tensor Products}

The frame concept was introduced by Duffin and Schaeffer in [22] while studying some problems in nonharmonic Fourier series; some years later it was revived by Daubechies et al. in [23]. Nowadays, frames have become a tool in pure and applied mathematics, computer science, physics, and engineering used to derive redundant, yet stable decompositions of a signal for analysis or transmission, while also promoting sparse expansions. Recall that a sequence $\left\{x_{n}\right\}$ is a frame for a separable Hilbert space $\mathscr{H}$ if there exist two constants $A, B>$ 0 (frame bounds) such that

$$
A\|x\|^{2} \leq \sum_{n}\left|\left\langle x, x_{n}\right\rangle\right|^{2} \leq B\|x\|^{2} \quad \forall x \in \mathscr{H} .
$$

A sequence satisfying only the right-hand inequality is said to be a Bessel sequence for $\mathscr{H}$. Given a frame $\left\{x_{n}\right\}$ for $\mathscr{H}$ the representation property of any vector $x \in \mathscr{H}$ as a series $x=\sum_{n} c_{n} x_{n}$ is retained, but, unlike the case of Riesz bases, the uniqueness of this representation (for overcomplete frames) is sacrificed. Suitable frame coefficients $c_{n}$ which depend continuously and linearly on $x$ are obtained by using dual frames $\left\{y_{n}\right\}$ of $\left\{x_{n}\right\}$; that is, $\left\{y_{n}\right\}$ is another frame for $\mathscr{H}$ such that for each $x \in \mathscr{H}$

$$
x=\sum_{n}\left\langle x, y_{n}\right\rangle x_{n}=\sum_{n}\left\langle x, x_{n}\right\rangle y_{n} \quad \text { in } \mathscr{H} .
$$

Recall that a Riesz basis in a separable Hilbert space $\mathscr{H}$ is the image of an orthonormal basis by means of a boundedly invertible operator; it is a particular case of frame: the socalled exact frame. Any Riesz basis $\left\{x_{n}\right\}$ has a unique biorthonormal (dual) Riesz basis $\left\{y_{n}\right\}$; that is, $\left\langle x_{n}, y_{m}\right\rangle_{\mathscr{H}}=\delta_{n, m}$, such that expansion (6) holds for every $x \in \mathscr{H}$. A Riesz sequence is a Riesz basis for its closed span. For more details on frames and Riesz bases theory see, for instance, the monograph [8] and references therein; see also [24] for finite frames.

Traditionally, frames were used in signal and image processing, nonharmonic analysis, data compression, and sampling theory, but nowadays frame theory plays also a fundamental role in a wide variety of problems in both pure and applied mathematics, computer science, physics, and engineering. The redundancy of frames, which gives flexibility and robustness, is the key to their significance for applications; see, for instance, the nice introduction in Chapter 1 of [24] and the references therein.

Next we briefly recall some basic facts about tensor products of Hilbert spaces which will be useful in the current work. Let $\mathscr{H}_{1}$ and $\mathscr{H}_{2}$ be two Hilbert spaces. Among the different ways of constructing tensor product spaces we adopt the model proposed in [25]. There, the tensor product $\mathscr{H}_{1} \otimes$ $\mathscr{H}_{2}$ is defined as the space of all antilinear maps $A: \mathscr{H}_{2} \rightarrow$ $\mathscr{H}_{1}$ such that $\sum_{i}\left\|A e_{i}\right\|^{2}<\infty$ for some orthonormal basis of $\left\{e_{i}\right\}_{i}$ of $\mathscr{H}_{2}$.

As for every $A, B \in \mathscr{H}_{1} \otimes \mathscr{H}_{2}$ the series $\sum_{i}\left\|A e_{i}\right\|^{2}$ and $\sum_{i}\left\langle A e_{i}, B e_{i}\right\rangle$ are independent of the orthonormal basis $\left\{e_{i}\right\}_{i}$ for $\mathscr{H}_{2}$; then $\mathscr{H}_{1} \otimes \mathscr{H}_{2}$ can be turned into an inner product space by defining the norm $\|A\|^{2}=\sum_{i}\left\|A e_{i}\right\|^{2}$ (and the associated inner product $\left.\langle A, B\rangle=\sum_{i}\left\langle A e_{i}, B e_{i}\right\rangle\right)$. Indeed $\mathscr{H}_{1} \otimes \mathscr{H}_{2}$ endowed with this inner product becomes a Hilbert space.

If $u \in \mathscr{H}_{1}$ and $v \in \mathscr{H}_{2}$ the tensor product $u \otimes v \in \mathscr{H}_{1} \otimes$ $\mathscr{H}_{2}$ is defined to be the rank one map such that $(u \otimes v)(w)=$ $\langle v, w\rangle u$ for every $w \in \mathscr{H}_{2}$.

Let $\mathscr{B}\left(\mathscr{H}_{1}\right), \mathscr{B}\left(\mathscr{H}_{2}\right)$, and $\mathscr{B}\left(\mathscr{H}_{1} \otimes \mathscr{H}_{2}\right)$ denote the spaces of all bounded linear operators on $\mathscr{H}_{1}, \mathscr{H}_{2}$, and $\mathscr{H}_{1} \otimes \mathscr{H}_{2}$, respectively. If $S \in \mathscr{B}\left(\mathscr{H}_{1}\right)$ and $T \in \mathscr{B}\left(\mathscr{H}_{2}\right)$ the tensor product $S \otimes T \in \mathscr{B}\left(\mathscr{H}_{1} \otimes \mathscr{H}_{2}\right)$ is defined to be the bounded 
linear operator on $\mathscr{H}_{1} \otimes \mathscr{H}_{2}$ such that $(S \otimes T)(A)=S A T^{*}$ for every $A \in \mathscr{H}_{1} \otimes \mathscr{H}_{2}$.

For further information on tensor products of Hilbert spaces see [25-28]. In these sources the following results for tensor products needed in the sequel are to be found.

(i) $\|u \otimes v\|=\|u\|\|v\|$ and $\left\langle u \otimes v, u^{\prime} \otimes v^{\prime}\right\rangle=\left\langle u, u^{\prime}\right\rangle\left\langle v, v^{\prime}\right\rangle$ for any $u, u^{\prime} \in \mathscr{H}_{1}$ and $v, v^{\prime} \in \mathscr{H}_{2}$.

(ii) $\|S \otimes T\|=\|S\|\|T\|$ and $(S \otimes T)(u \otimes v)=S u \otimes T v$ for any $S \in \mathscr{B}\left(\mathscr{H}_{1}\right), T \in \mathscr{B}\left(\mathscr{H}_{2}\right), u \in \mathscr{H}_{1}$, and $v \in \mathscr{H}_{2}$.

(iii) The linear span of $\left\{u \otimes v: u \in \mathscr{H}_{1}, v \in \mathscr{H}_{2}\right\}$ is dense in $\mathscr{H}_{1} \otimes \mathscr{H}_{2}$.

(iv) The tensor product of two orthonormal bases is an orthonormal basis.

(v) The operator $S \otimes T$ is invertible in $\mathscr{B}\left(\mathscr{H}_{1} \otimes \mathscr{H}_{2}\right)$ if and only if each operator, $S$ and $T$, is invertible in $\mathscr{B}\left(\mathscr{H}_{1}\right)$ and $\mathscr{B}\left(\mathscr{H}_{2}\right)$, respectively.

(vi) The tensor product of two sequences is a Riesz basis for $\mathscr{H}_{1} \otimes \mathscr{H}_{2}$ if and only if each sequence is a Riesz basis for its corresponding Hilbert space.

(vii) The tensor product of two Bessel sequences is a Bessel sequence for the corresponding Hilbert space.

(viii) The tensor product of two sequences is a frame for $\mathscr{H}_{1} \otimes \mathscr{H}_{2}$ if and only if each sequence is a frame for its corresponding Hilbert space.

Note that the fact that $\mathscr{H}_{1} \otimes \mathscr{H}_{2}$ is the completion of the linear span of $\mathscr{D}:=\left\{u \otimes v: u \in \mathscr{H}_{1}, v \in \mathscr{H}_{2}\right\}$ yields that any operator of $\mathscr{B}\left(\mathscr{H}_{1} \otimes \mathscr{H}_{2}\right)$, in particular the tensor product $S \otimes T \in \mathscr{B}\left(\mathscr{H}_{1} \otimes \mathscr{H}_{2}\right)$, is determined by its values on $\mathscr{D}$.

Finally, let $(X, \mu)$ and $(Y, \nu)$ denote two $\sigma$-finite measure spaces, and then we have that $L^{2}(\mu) \otimes L^{2}(\nu)=L^{2}(\mu \times \nu)$ via the identification $(f \otimes g)(x, y)=f(x) g(y)$.

\section{Infinite-Finite Generators Case}

Let $\mathscr{H}_{1}$ and $\mathscr{H}_{2}$ be two separable Hilbert spaces and $U$ : $\mathscr{H}_{1} \rightarrow \mathscr{H}_{1}$ and $V: \mathscr{H}_{2} \rightarrow \mathscr{H}_{2}$ two unitary operators. Consider two elements $a \in \mathscr{H}_{1}$ and $b \in \mathscr{H}_{2}$ such that the sequence $\left\{U^{n} a\right\}_{n \in \mathbb{Z}}$ forms a Riesz sequence in $\mathscr{H}_{1}$ (see $[2$, Theorem 2.1] for a necessary and sufficient condition), and there exists an $N \in \mathbb{N}$ such that $V^{N} b=b$, being the set $\left\{b, V b, V^{2} b, \ldots, V^{N-1} b\right\}$ linearly independent in $\mathscr{H}_{2}$ (see $[3$, Proposition 1] for a necessary and sufficient condition). In the tensor product Hilbert space $\mathscr{H}_{1} \otimes \mathscr{H}_{2}$ we consider the closed subspace

$$
\mathscr{A}_{a, b}:=\overline{\operatorname{span}}_{\mathscr{H}_{1} \otimes \mathscr{H}_{2}}\left\{U^{n} a \otimes V^{p} b\right\}_{n \in \mathbb{Z} ; p=0,1, \ldots, N-1} .
$$

Since the sequence $\left\{U^{n} a \otimes V^{p} b\right\}_{n \in \mathbb{Z} ; p=0,1, \ldots, N-1}$ is a Riesz basis for the tensor product $\mathscr{A}_{a} \otimes \mathscr{A}_{b}$ of the $U$-invariant subspace $\mathscr{A}_{a}=\left\{\sum_{n \in \mathbb{Z}} a_{n} U^{n} a:\left\{a_{n}\right\} \in \ell^{2}(\mathbb{Z})\right\}$ of $\mathscr{H}_{1}$ and the $V$-invariant subspace $\mathscr{A}_{b}=\left\{\sum_{p=0}^{N-1} b_{p} V^{p} b: b_{p} \in \mathbb{C}\right\}$ of $\mathscr{H}_{2}$ we deduce that $\mathscr{A}_{a, b}=\mathscr{A}_{a} \otimes \mathscr{A}_{b}$, and it can be described as

$$
\begin{aligned}
\mathscr{A}_{a, b} & =\left\{\sum_{n \in \mathbb{Z}} \sum_{p=0}^{N-1} a_{n p} U^{n} a \otimes V^{p} b:\left\{a_{n p}\right\}_{n} \in \ell^{2}(\mathbb{Z}), p\right. \\
& =0,1, \ldots, N-1\} .
\end{aligned}
$$

We will refer to the vectors $\{a, b\}$ as the infinite-finite generators of the subspace $\mathscr{A}_{a, b}$ in $\mathscr{H}_{1} \otimes \mathscr{H}_{2}$.

The Samples. Fix $S=s s^{\prime}$ elements $h_{j j^{\prime}}:=h_{j 1} \otimes h_{j^{\prime} 2} \in \mathscr{H}_{1} \otimes$ $\mathscr{H}_{2}$, where $j=1,2, \ldots, s$ and $j^{\prime}=1,2, \ldots, s^{\prime}$. Consider two sampling periods $r$ and $\bar{r}$, where $r \in \mathbb{N}$ and $\bar{r}$ is a divisor of $N$; denote $\ell:=N / \bar{r}$. For each $x \in \mathscr{A}_{a, b}$ we introduce the sequence of its generalized samples

$$
\left\{\mathscr{L}_{j j^{\prime}} x(r n, \bar{r} m)\right\}_{n \in \mathbb{Z} ; m=0,1, \ldots, \ell-1 ; j=1,2, \ldots, s ; j^{\prime}=1,2, \ldots, s^{\prime}}
$$

defined by

$$
\mathscr{L}_{j j^{\prime}} x(r n, \bar{r} m):=\left\langle x, U^{r n} h_{j 1} \otimes V^{\bar{r} m} h_{j^{\prime} 2}\right\rangle_{\mathscr{H}_{1} \otimes \mathscr{H}_{2}},
$$

where $n \in \mathbb{Z}, m=0,1, \ldots, \ell-1, j=1,2, \ldots, s$, and $j^{\prime}=$ $1,2, \ldots, s^{\prime}$. We will refer to any $\mathscr{L}_{j j^{\prime}}$ as a $U V$-system acting on the subspace $\mathscr{A}_{a, b}$.

The Isomorphism $\mathscr{T}_{a, b}^{U V}$. Let $\ell_{N}^{2}(\mathbb{Z})$ denote the space of all $N$-periodic sequences in $\mathbb{C}$ with inner product $\langle\mathbf{x}, \mathbf{y}\rangle_{\ell_{N}^{2}}=$ $\sum_{p=0}^{N-1} x(p) \overline{y(p)}$ and its canonical basis as $\left\{\mathbf{e}_{p}\right\}_{p=0}^{N-1}$. This space is isomorphic to the euclidean space $\mathbb{C}^{N}$; along the paper we will identify sequences in $\ell_{N}^{2}(\mathbb{Z})$ with vectors in $\mathbb{C}^{N}$ : any vector in $\mathbb{C}^{N}$ defines the terms from 0 to $N-1$ of the corresponding sequence in $\ell_{N}^{2}(\mathbb{Z})$.

We introduce the isomorphism $\mathscr{T}_{a, b}^{U V}$ which maps the canonical orthonormal basis $\left\{e^{2 \pi i n x} \otimes \mathbf{e}_{p}\right\}_{n \in \mathbb{Z} ; p=0,1, \ldots, N-1}$ for the Hilbert space $L^{2}(0,1) \otimes \ell_{N}^{2}(\mathbb{Z})$ onto the Riesz basis $\left\{U^{n} a \otimes\right.$ $\left.V^{p} b\right\}_{n \in \mathbb{Z} ; p=0,1, \ldots, N-1}$ for $\mathscr{A}_{a, b}$. That is,

$$
\begin{aligned}
\mathscr{T}_{a, b}^{U V}: & L^{2}(0,1) \otimes \ell_{N}^{2}(\mathbb{Z}) \longrightarrow \mathscr{A}_{a, b} \\
& e^{2 \pi i n x} \otimes \mathbf{e}_{p} \longmapsto U^{n} a \otimes V^{p} b,
\end{aligned}
$$

where $n \in \mathbb{Z}$ and $p=0,1, \ldots, N-1$.

It is clear that $\mathscr{T}_{a, b}^{U V}=\mathscr{T}_{a}^{U} \otimes \mathscr{T}_{b, N}^{V}$, where $\mathscr{T}_{a}^{U}$ and $\mathscr{T}_{b, N}^{V}$ denote the isomorphisms

$$
\begin{aligned}
\mathscr{T}_{a}^{U}: & L^{2}(0,1) \longrightarrow \mathscr{A}_{a} \\
& e^{2 \pi i n x} \longmapsto U^{n} a, \\
\mathscr{T}_{b, N}^{V}: & e_{N}^{2}(\mathbb{Z}) \longrightarrow \mathscr{A}_{b} \\
& \mathbf{e}_{p} \longmapsto V^{p} b .
\end{aligned}
$$

The following shifting property will be crucial later in obtaining our sampling formulas. 
Lemma 1. Let $g \in L^{2}(0,1)$ and $\mathbb{T}_{0}=(T(0), T(1), \ldots, T(N-$ 1)) $\in \ell_{N}^{2}(\mathbb{Z})$. For $M \in \mathbb{Z}$ and $1 \leq q \leq N-1$ the shifting property

$$
\begin{aligned}
& \mathscr{T}_{a, b}^{U V}\left(g(x) e^{2 \pi i M x} \otimes \mathbb{T}_{N-q}\right) \\
& =\left(U^{M} \otimes V^{q}\right) \mathscr{T}_{a, b}^{U V}\left(g \otimes \mathbb{T}_{0}\right)
\end{aligned}
$$

holds, where $\mathbb{T}_{N-q}$ denotes the sequence obtained from $\mathbb{T}_{0}$ as

$$
\begin{gathered}
\mathbb{\mathbb { T }}_{N-q}:=(T(N-q), T(N-q+1), \ldots, \\
T(N-q+N-1)) .
\end{gathered}
$$

Proof. Indeed, having in mind the shifting properties: $\mathscr{T}_{a}^{U}\left(g(x) e^{2 \pi i M x}\right)=U^{M}\left(\mathscr{T}_{a}^{U} g\right)$ for $\mathscr{T}_{a}^{U}$ (see [2, Eq. (2.1)]) and $\mathscr{T}_{b, N}^{V}\left(\mathbb{T}_{N-q}\right)=V^{q}\left(\mathscr{T}_{b, N}^{V} \mathbb{\mathbb { T }}_{0}\right)$ for $\mathscr{T}_{b, N}^{V}$ (see [3, Prop. 2]), and the properties of the tensor product of operators, we have

$$
\begin{aligned}
\mathscr{T}_{a, b}^{U V}\left(g(x) e^{2 \pi i M x} \otimes \mathbb{T}_{N-q}\right) \\
=\mathscr{T}_{a}^{U}\left(g(x) e^{2 \pi i M x}\right) \otimes \mathscr{T}_{b, N}^{V}\left(\mathbb{T}_{N-q}\right) \\
=U^{M}\left(\mathscr{T}_{a}^{U} g\right) \otimes V^{q}\left(\mathscr{T}_{b, N}^{V} \mathbb{\mathbb { T }}_{0}\right) \\
=\left(U^{M} \otimes V^{q}\right)\left(\mathscr{T}_{a}^{U} g \otimes \mathscr{T}_{b, N}^{V} \mathbb{T}_{0}\right) \\
=\left(U^{M} \otimes V^{q}\right) \mathscr{T}_{a, b}^{U V}\left(g \otimes \mathbb{T}_{0}\right) .
\end{aligned}
$$

An Expression for the Samples. A suitable expression for the samples given in (10) will allow us to obtain the reconstruction conditions of any $x$ in the subspace $\mathscr{A}_{a, b}$ from the sequence of its samples (10). Indeed, for $x=\sum_{k \in \mathbb{Z}} \sum_{p=0}^{N-1} a_{k p} U^{k} a \otimes V^{p} b$ in $\mathscr{A}_{a, b}$ we have

$$
\begin{aligned}
& \mathscr{L}_{j j^{\prime}} x(r n, \bar{r} m) \\
& =\left\langle\sum_{k \in \mathbb{Z}} \sum_{p=0}^{N-1} a_{k p} U^{k} a \otimes V^{p} b, U^{r n} h_{j 1} \otimes V^{\bar{r} m} h_{j^{\prime} 2}\right\rangle_{\mathscr{H}_{1} \otimes \mathscr{H}_{2}} \\
& =\sum_{k \in \mathbb{Z}} \sum_{p=0}^{N-1} a_{k p}\left\langle U^{k} a \otimes V^{p} b, U^{r n} h_{j 1} \otimes V^{\bar{r} m} h_{j^{\prime} 2}\right\rangle_{\mathscr{H}_{1} \otimes \mathscr{H}_{2}} .
\end{aligned}
$$

Now, consider $F:=\sum_{k \in \mathbb{Z}} \sum_{p=0}^{N-1} a_{k p} e^{2 \pi i k x} \otimes \mathbf{e}_{p}$, which is the element in $L^{2}(0,1) \otimes \ell_{N}^{2}(\mathbb{Z})$ such that $\mathscr{T}_{a, b}^{U V} F=x$. Thus, Plancherel identity for orthonormal bases gives

$$
\begin{gathered}
\mathscr{L}_{j j^{\prime}} x(r n, \bar{r} m)=\left\langle F, \sum_{k \in \mathbb{Z}} \sum_{p=0}^{N-1}\left\langle\overline{a, U^{r n-k} h_{j 1}}\right\rangle_{\mathscr{H}_{1}}\right. \\
\left.\cdot\left\langle\overline{b, V^{\bar{r} m-p} h_{j^{\prime} 2}}\right\rangle_{\mathscr{H}_{2}} e^{2 \pi i k x} \otimes \mathbf{e}_{p}\right\rangle_{L^{2}(0,1) \otimes \ell_{N}^{2}(\mathbb{Z})}
\end{gathered}
$$

$$
\begin{aligned}
& =\left\langle F,\left(\sum_{k \in \mathbb{Z}}\left\langle\overline{a, U^{r n-k} h_{j 1}}\right\rangle_{\mathscr{H}_{1}} e^{2 \pi i k x}\right)\right. \\
& \left.\otimes\left(\sum_{p=0}^{N-1}\left\langle\overline{\left\langle, V^{\bar{r} m-p} h_{j^{\prime} 2}\right.}\right\rangle_{\mathscr{H}_{2}} \mathbf{e}_{p}\right)\right\rangle_{L^{2}(0,1) \otimes \ell_{N}^{2}(\mathbb{Z})} .
\end{aligned}
$$

That is, for $n \in \mathbb{Z}, m=0,1, \ldots, \ell-1, j=1,2, \ldots, s$, and $j^{\prime}=1,2, \ldots, s^{\prime}$ we have got the following expression for the samples:

$$
\begin{aligned}
& \mathscr{L}_{j j^{\prime}} x(r n, \bar{r} m) \\
& =\left\langle F, \overline{g_{j}(x)} e^{2 \pi i r n x} \otimes \mathbb{G}_{j^{\prime}, m}\right\rangle_{L^{2}(0,1) \otimes \ell_{N}^{2}(\mathbb{Z})},
\end{aligned}
$$

where $\mathscr{T}_{a, b}^{U V} F=x$, the functions

$$
g_{j}(x):=\sum_{k \in \mathbb{Z}}\left\langle a, U^{k} h_{j 1}\right\rangle_{\mathscr{H}_{1}} e^{2 \pi i k x}, \quad j=1,2, \ldots, s
$$

belong to $L^{2}(0,1)$ (recall that $\left\{U^{n} a\right\}_{n \in \mathbb{Z}}$ is a Riesz sequence for $\left.\mathscr{H}_{1}\right)$, and

$$
\begin{aligned}
\mathbb{G}_{j^{\prime}, m} & :=\sum_{p=0}^{N-1}\left\langle\overline{\left\langle, V^{\bar{r} m-p} h_{j^{\prime} 2}\right.}\right\rangle_{\mathscr{H}_{2}} \mathbf{e}_{p} \\
& =\sum_{p=0}^{N-1} \overline{R_{b, h_{j^{\prime} 2}}(N+p-\bar{r} m)} \mathbf{e}_{p}, \quad j^{\prime}=1,2, \ldots, s^{\prime},
\end{aligned}
$$

where

$$
R_{b, h_{j^{\prime} 2}}(q):=\left\langle V^{q} b, h_{j^{\prime} 2}\right\rangle_{\mathscr{H}_{2}}, \quad q \in \mathbb{Z},
$$

denotes the ( $N$-periodic) cross-covariance sequence between the sequences $\left\{V^{q} b\right\}_{q \in \mathbb{Z}}$ and $\left\{V^{q} h_{j^{\prime} 2}\right\}_{q \in \mathbb{Z}}$ in $\mathscr{H}_{2}$ (see [29]). Thus, we deduce the following result.

Proposition 2. Any element $x \in \mathscr{A}_{a, b}$ can be recovered from the sequence of its samples $\left\{\mathscr{L}_{j j^{\prime}} x(r n\right.$, $\bar{r} m)\}_{n \in \mathbb{Z} ; m=0,1, \ldots, \ell-1 ; j=1,2, \ldots, s ; j^{\prime}=1,2, \ldots, s^{\prime}}$ in a stable way (by means of a frame expansion) if and only if the sequence $\left\{\overline{g_{j}(x)} e^{2 \pi i r n x} \otimes \mathbb{G}_{j^{\prime}, m}\right\}_{n \in \mathbb{Z} ; m=0,1, \ldots, \ell-1 ; j=1,2, \ldots, s ; j^{\prime}=1,2, \ldots, s^{\prime}}$ is a frame for $L^{2}(0,1) \otimes \ell_{N}^{2}(\mathbb{Z})$.

Having in mind that a tensor product of two sequences is a frame in the tensor product if and only if the respective factors are frames, we only need a characterization of the sequences $\left\{\overline{g_{j}(x)} e^{2 \pi i r n x}\right\}_{n \in \mathbb{Z} ; j=1,2, \ldots, s}$ and $\left\{\mathbb{G}_{j^{\prime}, m}\right\}_{m=0,1, \ldots, \ell-1 ; j^{\prime}=1,2, \ldots, s^{\prime}}$ as frames for $L^{2}(0,1)$ and $\ell_{N}^{2}(\mathbb{Z})$, respectively. This study has been done in $[3,10]$, respectively. 
(i) For the first one, consider the $s \times r$ matrix of functions in $L^{2}(0,1)$

$$
\begin{aligned}
& \mathbf{G}(x) \\
& :=\left(\begin{array}{cccc}
g_{1}(x) & g_{1}\left(x+\frac{1}{r}\right) & \cdots & g_{1}\left(x+\frac{r-1}{r}\right) \\
g_{2}(x) & g_{2}\left(x+\frac{1}{r}\right) & \cdots & g_{2}\left(x+\frac{r-1}{r}\right) \\
\vdots & \vdots & \vdots \\
g_{s}(x) & g_{s}\left(x+\frac{1}{r}\right) & \cdots & g_{s}\left(x+\frac{r-1}{r}\right)
\end{array}\right) \\
& =\left(\begin{array}{ccc}
\left.g_{j}\left(x+\frac{k-1}{r}\right)\right)_{j=1,2, \ldots, s ; k=1,2, \ldots, r}
\end{array}\right.
\end{aligned}
$$

and its related constants

$$
\begin{aligned}
& \alpha_{\mathbf{G}}:=\underset{x \in(0,1 / r)}{\operatorname{essinf}} \lambda_{\min }\left[\mathbf{G}^{*}(x) \mathbf{G}(x)\right], \\
& \beta_{\mathbf{G}}:=\underset{x \in(0,1 / r)}{\operatorname{ess} \sup _{\max }} \lambda_{\operatorname{mo}}\left[\mathbf{G}^{*}(x) \mathbf{G}(x)\right],
\end{aligned}
$$

where $\mathbf{G}^{*}(x)$ denotes the transpose conjugate of the matrix $\mathrm{G}(x)$, and $\lambda_{\min }$ (resp., $\lambda_{\max }$ ) the smallest (resp., the largest) eigenvalue of the positive semidefinite matrix $\mathbf{G}^{*}(x) \mathbf{G}(x)$. Observe that $0 \leq \alpha_{\mathrm{G}} \leq \beta_{\mathrm{G}} \leq \infty$. Notice that in the definition of the matrix $\mathbf{G}(x)$ we are considering 1-periodic extensions of the involved functions $g_{j}, j=1,2, \ldots, s$.
A complete characterization of the sequence $\left\{\overline{g_{j}(x)} e^{2 \pi i r n x}\right\}_{n \in \mathbb{Z} ; j=1,2, \ldots, s}$ as a frame or a Riesz basis for $L^{2}(0,1)$ is given in the next lemma (see [10, Lemma 3] or [11, Lemma 2] for the proof).

Lemma 3. For the functions $g_{j} \in L^{2}(0,1), j=1,2, \ldots, s$, consider the associated matrix $\mathbf{G}(x)$ given in (22). Then, the following results hold:

(a) The sequence $\left\{\overline{g_{j}(x)} e^{2 \pi i r n x}\right\}_{n \in \mathbb{Z} ; j=1,2, \ldots, s}$ is a complete system for $L^{2}(0,1)$ if and only if the rank of the matrix $\mathbf{G}(x)$ is $r$ a.e. in $(0,1 / r)$.

(b) The sequence $\left\{\overline{g_{j}(x)} e^{2 \pi i r n x}\right\}_{n \in \mathbb{Z} ; j=1,2, \ldots, s}$ is a Bessel sequence for $L^{2}(0,1)$ if and only if $g_{j} \in L^{\infty}(0,1)$ (or equivalently $\left.\beta_{\mathrm{G}}<\infty\right)$. In this case, the optimal Bessel bound is $\beta_{\mathrm{G}} / r$.

(c) The sequence $\left\{\overline{g_{j}(x)} e^{2 \pi i r n x}\right\}_{n \in \mathbb{Z} ; j=1,2, \ldots, s}$ is a frame for $L^{2}(0,1)$ if and only if $0<\alpha_{\mathbf{G}} \leq \beta_{\mathbf{G}}<\infty$. In this case, the optimal frame bounds are $\alpha_{\mathrm{G}} / r$ and $\beta_{\mathrm{G}} / r$.

(d) The sequence $\left\{\overline{g_{j}(x)} e^{2 \pi i r n x}\right\}_{n \in \mathbb{Z} ; j=1,2, \ldots, s}$ is a Riesz basis for $L^{2}(0,1)$ if and only if it is a frame and $s=r$.

(ii) For the second one, consider the $s^{\prime} \ell \times N$ matrix of cross-covariances

$$
\mathbf{R}_{\mathbf{b}, \mathbf{h}_{2}}:=\left(\begin{array}{c}
\mathbf{R}_{\mathbf{b}, \mathbf{h}_{12}} \\
\mathbf{R}_{\mathbf{b}, \mathbf{h}_{22}} \\
\vdots \\
\mathbf{R}_{\mathbf{b}, \mathbf{h}_{\mathbf{s}^{\prime} 2}}
\end{array}\right),
$$

where each $\ell \times N$ block $\mathbf{R}_{\mathbf{b}, \mathbf{h}_{\mathbf{j}^{\prime}{ }^{\prime}}}, j^{\prime}=1,2, \ldots, s^{\prime}$, is given by

$$
\mathbf{R}_{\mathbf{b}, h_{\mathbf{j}^{\prime} 2}}=\left(\begin{array}{cccc}
R_{b, h_{j^{\prime} 2}}(0) & R_{b, h_{j^{\prime} 2}}(1) & \cdots & R_{b, h_{j^{\prime} 2}}(N-1) \\
R_{b, h_{j^{\prime} 2}}(N-\bar{r}) & R_{b, h_{j^{\prime} 2}}(N-\bar{r}+1) & \cdots & R_{b, h_{j^{\prime} 2}}(2 N-\bar{r}-1) \\
\vdots & \vdots & \ddots & \vdots \\
R_{b, h_{j^{\prime} 2}}(N-\bar{r}(\ell-1)) & R_{b, h_{j^{\prime} 2}}(N-\bar{r}(\ell-1)+1) & \cdots & R_{b, h_{j^{\prime} 2}}(N-\bar{r}(\ell-1)+N-1)
\end{array}\right) .
$$

In [3] the following lemma was proved.

Lemma 4. The sequence $\left\{\mathbb{G}_{j^{\prime}, m}\right\}_{m=0,1, \ldots, \ell-1 ; j^{\prime}=1,2, \ldots, s^{\prime}}$ is a frame for $\ell_{N}^{2}(\mathbb{Z})$ (or equivalently, a spanning set since we are in finite dimension) for $\ell_{N}^{2}(\mathbb{Z})$ if and only if rank $\mathbf{R}_{\mathbf{b}, \mathbf{h}_{2}}=N$.

Notice that, necessarily, $s \geq r$ and $s^{\prime} \geq \bar{r}$ and, consequently, the number $S=s s^{\prime}$ of needed $U V$-systems $\mathscr{L}_{j j^{\prime}}$ must be $S \geq r \bar{r}$.

3.1. The Sampling Result. In case that $\left\{\overline{g_{j}(x)} e^{2 \pi i r n x} \otimes\right.$ $\left.\mathbb{G}_{j^{\prime}, m}\right\}_{n \in \mathbb{Z} ; m=0,1, \ldots, \ell-1 ; j=1,2, \ldots, s ; j^{\prime}=1,2, \ldots, s^{\prime}}$ is a frame for $L^{2}(0,1) \otimes$ $\ell_{N}^{2}(\mathbb{Z})$ we need to describe the family of appropriate (for sampling purposes) dual frames. In $[3,10]$ suitable dual frames of the frames $\left\{\overline{g_{j}(x)} e^{2 \pi i r n x}\right\}_{n \in \mathbb{Z} ; j=1,2, \ldots, s}$ for $L^{2}(0,1)$ and $\left\{\mathbb{G}_{j^{\prime}, m}\right\}_{m=0,1, \ldots, \ell-1 ; j^{\prime}=1,2, \ldots, s^{\prime}}$ for $\ell_{N}^{2}(\mathbb{Z})$, respectively, have been obtained. For a notational and reading ease we recall them.

(i) For the first case, choose functions $h_{j}$ in $L^{\infty}(0,1), j=$ $1,2, \ldots, s$, such that

$$
\begin{array}{r}
{\left[h_{1}(x), h_{2}(x), \ldots, h_{s}(x)\right] \mathbf{G}(x)=[1,0, \ldots, 0]} \\
\text { a.e. in }(0,1) .
\end{array}
$$

It was proven in [10] that the sequence $\left\{r h_{j}(x) e^{2 \pi i r n x}\right\}_{n \in \mathbb{Z} ; j=1,2, \ldots, s}$ is a dual frame of the frame $\left\{\overline{g_{j}(x)} e^{2 \pi i r n x}\right\}_{n \in \mathbb{Z} ; j=1,2, \ldots, s}$ in $L^{2}(0,1)$. 
All the possible choices in (26) are given by the first row of the $r \times s$ matrices given by

$$
\mathbf{H}_{\mathbf{U}}(x):=\mathbf{G}^{\dagger}(x)+\mathbf{U}(x)\left[\mathbf{I}_{s}-\mathbf{G}(x) \mathbf{G}^{\dagger}(x)\right],
$$

where $\mathbf{G}^{\dagger}(x)$ denotes the Moore-Penrose pseudoinverse of $\mathbf{G}(x)$ given by

$$
\mathbf{G}^{\dagger}(x)=\left[\mathbf{G}^{*}(x) \mathbf{G}(x)\right]^{-1} \mathbf{G}^{*}(x),
$$

$\mathbf{U}(x)$ is any $r \times s$ matrix with entries in $L^{\infty}(0,1)$, and $\mathbf{I}_{s}$ is the identity matrix of order $s$ (see [30]). Notice that the entries of $\mathbf{G}^{\dagger}(x)$ are essentially bounded in $(0,1)$ since the functions $g_{j}, j=1,2, \ldots, s$, and $\operatorname{det}^{-1}\left[\mathbf{G}^{*}(x) \mathbf{G}(x)\right]$ are essentially bounded in $(0,1)$.

(ii) For the second case, the $N$-periodic extensions of the columns $\left\{\mathbb{H}_{j^{\prime}, m}\right\}_{m=0,1, \ldots, \ell-1 ; j^{\prime}=1,2, \ldots, s^{\prime}}$ of a left-inverse $\mathbf{H}_{\mathbf{S}}$ of the $s^{\prime} \ell \times N$ matrix $\mathbf{R}_{\mathbf{b}, \mathbf{h}_{2}}$, written as

$$
\mathbf{H}_{\mathbf{S}}=\left(\begin{array}{cccccccccc}
\vdots & \vdots & \vdots & \vdots & \vdots & \vdots & \vdots & \vdots & \vdots & \vdots \\
\mathbb{H}_{1,0} & \cdots & \mathbb{W}_{1, \ell-1} & \mathbb{H}_{2,0} & \cdots & \mathbb{H}_{2, \ell-1} & \cdots & \mathbb{W}_{s^{\prime}, 0} & \cdots & \mathbb{U}_{\mathcal{s}^{\prime}, \ell-1} \\
\vdots & \vdots & \vdots & \vdots & \vdots & \vdots & \vdots & \vdots & \vdots & \vdots
\end{array}\right),
$$

and constructed from a $\bar{r} \times s^{\prime} \ell$ matrix $\mathbf{S}$ such that

$$
\mathbf{S R}_{\mathbf{b}, \mathbf{h}_{\mathbf{2}}}=\left(\mathbf{I}_{\bar{r}}, \mathbf{O}_{\bar{r} \times(N-\bar{r})}\right)
$$

as in [3, Section 3.1], form an appropriate dual frame of $\left\{\mathbb{G}_{j^{\prime}, m}\right\}_{m=0,1, \ldots, \ell-1 ; j^{\prime}=1,2, \ldots, s^{\prime}}$ in $\ell_{N}^{2}(\mathbb{Z})$ (see [3] for the details about the construction of $\mathbf{H}_{\mathrm{S}}$ ).

All the possible $\bar{r} \times s^{\prime} \ell$ matrices $\mathbf{S}$ satisfying (30) are given by the first $\bar{r}$ rows of any left-inverse $\mathbf{H}$ of the matrix $\mathbf{R}_{\mathbf{b}, \mathbf{h}_{2}}$. All these left-inverses can be expressed as

$$
\mathbf{H}=\mathbf{R}_{\mathbf{b}, \mathbf{h}_{2}}^{\dagger}+\mathbf{U}\left[\mathbf{I}_{s^{\prime} \ell}-\mathbf{R}_{\mathbf{b}, \mathbf{h}_{2}} \mathbf{R}_{\mathbf{b}, \mathbf{h}_{2}}^{\dagger}\right],
$$

where $\mathbf{R}_{\mathbf{b}, \mathbf{h}_{\mathbf{2}}}^{\dagger}$ denotes the Moore-Penrose pseudoinverse of $\mathbf{R}_{\mathbf{b}, \mathbf{h}_{\mathbf{2}}}$ and $\mathbf{U}$ is any arbitrary $N \times s^{\prime} \ell$ matrix.

Finally, one deduce that the sequence $\left\{r h_{j}(x) e^{2 \pi i r n x} \otimes\right.$ $\left.\mathbb{U}_{j^{\prime}, m}\right\}_{n \in \mathbb{Z} ; m=0,1, \ldots, \ell-1 ; j=1,2, \ldots, s ; j^{\prime}=1,2, \ldots, s^{\prime}}$ is a dual frame of $\left\{\overline{g_{j}(x)} e^{2 \pi i r n x} \otimes \mathbb{G}_{j^{\prime}, m}\right\}_{n \in \mathbb{Z} ; m=0,1, \ldots, \ell-1 ; j=1,2, \ldots, s ; j^{\prime}=1,2, \ldots, s^{\prime}} \quad$ in $L^{2}(0,1) \otimes \ell_{N}^{2}(\mathbb{Z})$. Indeed, we have the following lemma.

Lemma 5. Assume that $\left\{x_{n}^{1}\right\}$ and $\left\{y_{n}^{1}\right\}$ are dual frames in $\mathscr{H}_{1}$ and $\left\{x_{m}^{2}\right\}$ and $\left\{y_{m}^{2}\right\}$ are dual frames in $\mathscr{H}_{2}$. Then, the sequences $\left\{x_{n}^{1} \otimes x_{m}^{2}\right\}$ and $\left\{y_{n}^{1} \otimes y_{m}^{2}\right\}$ form a pair of dual frames in the tensor product $\mathscr{H}_{1} \otimes \mathscr{H}_{2}$.

Proof. Let $T_{N}^{k}$ and $G_{N}, k=1,2$ and $N \in \mathbb{N}$, be the bounded operators defined by

$$
\begin{aligned}
T_{N}^{k}: \quad \mathscr{H}_{k} \longrightarrow \mathscr{H}_{k} \\
h_{k} \longmapsto \sum_{n=-N}^{N}\left\langle h_{k}, y_{n}^{k}\right\rangle x_{n}^{k}, \\
G_{N}: \quad \mathscr{H}_{1} \otimes \mathscr{H}_{2} \longrightarrow \mathscr{H}_{1} \otimes \mathscr{H}_{2} \\
h \longmapsto \sum_{m, n=-N}^{N}\left\langle h, y_{n}^{1} \otimes y_{m}^{2}\right\rangle x_{n}^{1} \otimes y_{m}^{2} .
\end{aligned}
$$

The fact that $\left\{x_{n}^{k}\right\}$ and $\left\{y_{n}^{k}\right\}$ are dual frames in $\mathscr{H}_{k}$ implies that each sequence $\left\{T_{N}^{k}\right\}_{N \in \mathbb{N}}, k=1,2$, is a bounded sequence (in fact, $\left\|T_{N}^{k}\right\|^{2} \leq B_{x}^{k} B_{y}^{k}$ for every $N$, where $B_{x}^{k}$ and $B_{y}^{k}$ are the upper frame bounds of $\left\{x_{n}^{k}\right\}$ and $\left\{y_{n}^{k}\right\}$, resp.) which converges in the strong operator topology of $\mathscr{B}\left(\mathscr{H}_{k}\right)$ to the identity operator $I_{\mathscr{H}_{k}}$. Thus, Lemma 2.3 in [26] yields that $\left\{T_{N}^{1} \otimes T_{N}^{2}\right\}_{N \in \mathbb{N}}$ converges in the strong operator topology of $\mathscr{B}\left(\mathscr{H}_{1} \otimes \mathscr{H}_{2}\right)$ to $I_{\mathscr{H}_{1}} \otimes I_{\mathscr{H}_{2}}=I_{\mathscr{H}_{1} \otimes \mathscr{H}_{2}}$. Since

$$
\begin{aligned}
& \left(T_{N}^{1} \otimes T_{N}^{2}\right)\left(h_{1} \otimes h_{2}\right)=T_{N}^{1}\left(h_{1}\right) \otimes T_{N}^{2}\left(h_{2}\right) \\
& \quad=\left(\sum_{n=-N}^{N}\left\langle h_{1}, y_{n}^{1}\right\rangle x_{n}^{1}\right) \otimes\left(\sum_{m=-N}^{N}\left\langle h_{2}, y_{m}^{2}\right\rangle x_{m}^{2}\right) \\
& \quad=\sum_{m, n=-N}^{N}\left\langle h_{1}, y_{n}^{1}\right\rangle\left\langle h_{2}, y_{m}^{2}\right\rangle\left(x_{n}^{1} \otimes x_{m}^{2}\right) \\
& =G_{N}\left(h_{1} \otimes h_{2}\right)
\end{aligned}
$$

for every $N \in \mathbb{N}$ and every $h_{k} \in \mathscr{H}_{k}, k=1,2$, then, $\left(T_{N}^{1} \otimes\right.$ $\left.T_{N}^{2}\right)(h)=G_{N}(h)$ for every $h \in \mathscr{H}^{1} \otimes \mathscr{H}^{2}$ and every $N \in \mathbb{N}$. Hence $\left\{G_{N}\right\}_{N \in \mathbb{N}}$ converges in the strong operator topology of $\mathscr{B}\left(\mathscr{H}_{1} \otimes \mathscr{H}_{2}\right)$ to $I_{\mathscr{H}_{1} \otimes \mathscr{H}_{2}}$; that is,

$$
h=\sum_{m, n}\left\langle h, y_{n}^{1} \otimes y_{m}^{2}\right\rangle x_{n}^{1} \otimes y_{m}^{2}
$$

for every $h \in \mathscr{H}_{1} \otimes \mathscr{H}_{2}$, which concludes the proof.

As a consequence, for each $x \in \mathscr{A}_{a, b}$ there exists a unique $F \in L^{2}(0,1) \otimes \ell_{N}^{2}(\mathbb{Z})$ such that $\mathscr{T}_{a, b}^{U V} F=x$. This $F$ can be expressed as the frame expansion

F

$$
\begin{array}{r}
\sum_{j=1}^{s} \sum_{j^{\prime}=1}^{s^{\prime}} \sum_{n \in \mathbb{Z}} \sum_{m=0}^{\ell-1} \mathscr{L}_{j j^{\prime}} x(r n, \bar{r} m)\left(r h_{j}(x) e^{2 \pi i r n x} \otimes \mathbb{H}_{j^{\prime}, m}\right) \\
\operatorname{in~} L^{2}(0,1) \otimes \ell_{N}^{2}(\mathbb{Z}) .
\end{array}
$$

Then, applying the isomorphism $\mathscr{T}_{a, b}^{U V}$ and the shifting property in Lemma 1 (here it is the point where we are using 
the fact that the proposed dual frames are convenient for sampling purposes) one gets

$$
\begin{aligned}
x & \sum_{j=1}^{s} \sum_{j^{\prime}=1}^{s^{\prime}} \sum_{n \in \mathbb{Z}} \sum_{m=0}^{\ell-1} \mathscr{L}_{j j^{\prime}} x(r n, \bar{r} m) \mathscr{T}_{a, b}^{U V}\left(r h_{j}(x) e^{2 \pi i r n x} \otimes \mathbb{H}_{j^{\prime}, m}\right) \\
= & \sum_{j=1}^{s} \sum_{j^{\prime}=1}^{s^{\prime}} \sum_{n \in \mathbb{Z}} \sum_{m=0}^{\ell-1} \mathscr{L}_{j j^{\prime}} x(r n, \bar{r} m) U^{r n}\left(\mathscr{T}_{a}^{U} r h_{j}\right) \\
& \otimes V^{\bar{r} m}\left(\mathscr{T}_{b, N}^{V} \mathbb{H}_{j^{\prime}, 0}\right) \\
= & \sum_{j=1}^{s} \sum_{j^{\prime}=1}^{s^{\prime}} \sum_{n \in \mathbb{Z}} \sum_{m=0}^{\ell-1} \mathscr{L}_{j j^{\prime}} x(r n, \bar{r} m)\left(U^{r n} \otimes V^{\bar{r} m}\right)\left(c_{j} \otimes d_{j^{\prime}}\right),
\end{aligned}
$$

where $c_{j}=\mathscr{T}_{a}^{U}\left(r h_{j}\right) \in \mathscr{A}_{a}, j=1,2, \ldots, s$, and $d_{j^{\prime}}=$ $\mathscr{T}_{b, N}^{V}\left(\mathbb{H}_{j^{\prime}, 0}\right) \in \mathscr{A}_{b}, j^{\prime}=1,2, \ldots, s^{\prime}$.

Collecting the pieces we have obtained until now we prove the following result.

Theorem 6. Let $h_{j j^{\prime}}:=h_{j 1} \otimes h_{j^{\prime} 2} \in \mathscr{H}_{1} \otimes \mathscr{H}_{2}, j=1,2, \ldots, s$, $j^{\prime}=1,2, \ldots, s^{\prime}$, and let $\mathscr{L}_{j j^{\prime}}$ be the associated $U V$-system giving the samples of any $x \in \mathscr{A}_{a, b}$ as in (10), $j=1,2, \ldots, s$, $j^{\prime}=1,2, \ldots, s^{\prime}$. Assume that the function $g_{j}, j=1,2, \ldots, s$, given in (19) belongs to $L^{\infty}(0,1)$ or, equivalently, that $\beta_{\mathrm{G}}<$ $\infty$ for the associated $s \times r$ matrix $\mathbf{G}(x)$ defined in (22). The following statements are equivalent:

(a) $\alpha_{\mathbf{G}}>0$ and rank $\mathbf{R}_{\mathbf{b}, \mathbf{h}_{2}}=N$.

(b) There exist $S=s^{\prime}$ elements $c_{j} \otimes d_{j^{\prime}}$ in the subspace $\mathscr{A}_{a, b}$, $j=1,2, \ldots, s, j^{\prime}=1,2, \ldots, s^{\prime}$, such that the sequence $\left\{\left(U^{r n} \otimes V^{\bar{r} m}\right)\left(c_{j} \otimes d_{j^{\prime}}\right)\right\}_{n \in \mathbb{Z} ; m=0,1, \ldots, \ell-1 ; j=1,2, \ldots, s ; j^{\prime}=1,2, \ldots, s^{\prime}}$ is a frame for $\mathscr{A}_{a, b}$, and for any $x \in \mathscr{A}_{a, b}$ the expansion

$x$

$$
\begin{array}{r}
=\sum_{j=1}^{s} \sum_{j^{\prime}=1}^{s^{\prime}} \sum_{n \in \mathbb{Z}} \sum_{m=0}^{\ell-1} \mathscr{L}_{j j^{\prime}} x(r n, \bar{r} m)\left(U^{r n} \otimes V^{\bar{r} m}\right)\left(c_{j} \otimes d_{j^{\prime}}\right) \\
\text { in } \mathscr{H}_{1} \otimes \mathscr{H}_{2}
\end{array}
$$

holds.

(c) There exists a frame $\left\{C_{j, j^{\prime}, n, m}\right\}_{n \in \mathbb{Z} ; m=0,1, \ldots, \ell-1 ; j=1,2, \ldots, s ; j^{\prime}=1,2, \ldots, s^{\prime}} \quad$ for $\quad \mathscr{A}_{a, b}$ such that, for each $x \in \mathscr{A}_{a, b}$, the expansion

$$
x=\sum_{j=1}^{s} \sum_{j^{\prime}=1}^{s^{\prime}} \sum_{n \in \mathbb{Z}} \sum_{m=0}^{\ell-1} \mathscr{L}_{j j^{\prime}} x(r n, \bar{r} m) C_{j, j^{\prime}, n, m}
$$

$$
\text { in } \mathscr{H}_{1} \otimes \mathscr{H}_{2}
$$

holds.
Proof. It only remains to prove that condition (c) implies condition (a). Indeed, applying the isomorphism $\left(\mathscr{T}_{a, b}^{U V}\right)^{-1}$ to the expansion given in (c) one gets the frame expansion

$$
\begin{aligned}
&\left(\mathscr{T}_{a, b}^{U V}\right)^{-1}(x) \\
&=\sum_{j=1}^{s} \sum_{j^{\prime}=1}^{s^{\prime}} \sum_{n \in \mathbb{Z}} \sum_{m=0}^{\ell-1} \mathscr{L}_{j j^{\prime}} x(r n, \bar{r} m)\left(\mathscr{T}_{a, b}^{U V}\right)^{-1}\left(C_{j, j^{\prime}, n, m}\right) \\
& \operatorname{in~} L^{2}(0,1) \otimes \ell_{N}^{2}(\mathbb{Z}) .
\end{aligned}
$$

Having in mind (18) and that the sequence $\left\{\overline{g_{j}(x)} e^{2 \pi i r n x} \otimes\right.$ $\left.\mathbb{G}_{j^{\prime}, m}\right\}_{n \in \mathbb{Z} ; m=0,1, \ldots, \ell-1 ; j=1,2, \ldots, s ; j^{\prime}=1,2, \ldots, s^{\prime}}$ is a Bessel sequence for $L^{2}(0,1) \otimes \ell_{N}^{2}(\mathbb{Z})$, according to [8, Lemma 5.6.2], one gets that it is a frame for $L^{2}(0,1) \otimes \ell_{N}^{2}(\mathbb{Z})$. In particular, this implies, via Lemmas 3 and 4 , that $\alpha_{\mathbf{G}}>0$ and rank $\mathbf{R}_{\mathbf{b}, \mathbf{h}_{\mathbf{2}}}=N$.

In cases $s=r$ and $s^{\prime}=\bar{r}$ we are in the Riesz bases setting: see statement (d) in Lemma 3; moreover, the square matrix $\mathbf{R}_{\mathbf{b}, \mathbf{h}_{\mathbf{2}}}$ must be invertible (see [3, Corollary 4]). In fact, the following corollary holds.

Corollary 7. In addition to the hypotheses of the theorem above, assume that $s=r$ and $s^{\prime}=\bar{r}$. The following statements are equivalent:

(1) $\alpha_{\mathbf{G}}>0$ and the square matrix $\mathbf{R}_{\mathbf{b}, \mathbf{h}_{\mathbf{2}}}$ is invertible.

(2) There exist $r \bar{r}$ unique elements $c_{j} \otimes d_{j^{\prime}}$, $j=1,2, \ldots, r, j^{\prime}=1,2, \ldots, \bar{r}$, in the subspace $\mathscr{A}_{a, b}$ such that the sequence $\left\{\left(U^{r n} \otimes V^{\bar{r} m}\right)\left(c_{j} \otimes\right.\right.$ $\left.\left.d_{j^{\prime}}\right)\right\}_{n \in \mathbb{Z} ; m=0,1, \ldots, \ell-1 ; j=1,2, \ldots, r ; j^{\prime}=1,2, \ldots, \bar{r}}$ is a Riesz basis for $\mathscr{A}_{a, b}$, and the expansion of any $x \in \mathscr{A}_{a, b}$ with respect to this basis is

$x$

$$
\begin{array}{r}
=\sum_{j=1}^{r} \sum_{j^{\prime}=1}^{\bar{r}} \sum_{n \in \mathbb{Z}} \sum_{m=0}^{\ell-1} \mathscr{L}_{j j^{\prime}} x(r n, \bar{r} m)\left(U^{r n} \otimes V^{\bar{r} m}\right)\left(c_{j} \otimes d_{j^{\prime}}\right) \\
\text { in } \mathscr{H}_{1} \otimes \mathscr{H}_{2} .
\end{array}
$$

In case that the equivalent conditions are satisfied, the vectors $c_{j} \otimes d_{j^{\prime}}, j=1,2, \ldots, r, j^{\prime}=1,2, \ldots, \bar{r}$, satisfy the interpolation property

$$
\mathscr{L}_{k k^{\prime}}\left(c_{j} \otimes d_{j^{\prime}}\right)(r n, \bar{r} m)=\delta_{j, k} \delta_{j^{\prime}, k^{\prime}} \delta_{n, 0} \delta_{m, 0},
$$

whenever $n \in \mathbb{Z}, m=0,1, \ldots, \ell-1, j, k=1,2, \ldots, r$, and $j^{\prime}, k^{\prime}=1,2, \ldots, \bar{r}$.

Proof. The uniqueness of the expansion with respect to a Riesz basis gives the stated interpolation property (41).

A Representative Example. In $\mathscr{H}_{1}:=L^{2}(\mathbb{R})$ consider the shift operator $U: f(x) \mapsto f(x-1)$. Let $\varphi \in L^{2}(\mathbb{R})$ be a function such that the sequence $\{\varphi(x-n)\}_{n \in \mathbb{Z}}$ is a Riesz sequence in $L^{2}(\mathbb{R})$ (e.g., the function $\varphi$ may be a $B$-spline). 
Consider also the Hilbert space $\mathscr{H}_{2}:=L^{2}(0,1)$ of 1 -periodic functions and, for a fixed $N \in \mathbb{N}$, the unitary operator $V_{N}$ : $g(y) \mapsto g(y-1 / N)$. Let $\psi \in L^{2}(0,1)$ be a function such that the set $\left\{\psi, V_{N} \psi, \ldots, V_{N}^{N-1} \psi\right\}$ is linearly independent in $L^{2}(0,1)$; obviously $V_{N}^{N} \psi=\psi$ (e.g., choose a nonzero function $\psi \in L^{2}(0,1)$ taking the value 0 outside the interval $\left.(0,1 / N)\right)$.

In the tensor product $L^{2}(\mathbb{R}) \otimes L^{2}(0,1)=L^{2}(\mathbb{R} \times(0,1))$ consider the closed subspace $\mathscr{A}_{\varphi, \psi}:=\mathscr{A}_{\varphi} \otimes \mathscr{A}_{\psi}$, that is, the tensor product of the shift-invariant subspaces $\mathscr{A}_{\varphi}$ and $\mathscr{A}_{\psi}$ in $L^{2}(\mathbb{R})$. Given the functions $h_{j 1} \in L^{2}(\mathbb{R}), j=1,2, \ldots, s$, and $h_{j^{\prime} 2} \in L^{2}(0,1), j^{\prime}=1,2, \ldots, s^{\prime}$, and fixing the sampling periods $r \in \mathbb{N}$ and $\bar{r} \mid N$ (recall that $\ell=N / \bar{r}$ ), for each $f$ in $\mathscr{A}_{\varphi, \psi}$ we consider its samples defined by

$$
\begin{aligned}
& \mathscr{L}_{j j^{\prime}} f(r n, \bar{r} m) \\
& :=\int_{-\infty}^{\infty} \int_{0}^{1} f(x, y) \overline{h_{j 1}(x-r n)} \overline{h_{j^{\prime} 2}\left(y-\frac{m}{\ell}\right)} d x d y,
\end{aligned}
$$

where $n \in \mathbb{Z}$ and $m=0,1, \ldots, \ell-1$ (note that $\bar{r} m / N=m / \ell$ ).

Under the hypotheses in Theorem 6 there will exist functions $S_{j} \in \mathscr{A}_{\varphi}, j=1,2, \ldots, s$, and $\Theta_{j^{\prime}} \in \mathscr{A}_{\psi}, j^{\prime}=$ $1,2, \ldots, s^{\prime}$, such that for any $f \in \mathscr{A}_{\varphi, \psi}$ the sampling expansion (37) reads as follows:

$$
\begin{array}{r}
f(x, y) \\
=\sum_{j=1}^{s} \sum_{j^{\prime}=1}^{s^{\prime}} \sum_{n \in \mathbb{Z}} \sum_{m=0}^{\ell-1} \mathscr{L}_{j j^{\prime}} f(r n, \bar{r} m) S_{j}(x-r n) \Theta_{j^{\prime}}\left(y-\frac{m}{\ell}\right) \\
\text { in } L^{2}(\mathbb{R} \times(0,1)) .
\end{array}
$$

In this particular example, adding some mild hypotheses we can also derive pointwise convergence in the above sampling formula. Indeed, assuming that the generators $\varphi$ and $\psi$ are continuous functions on $\mathbb{R}$ such that $\sum_{n \in \mathbb{Z}} \mid \varphi(x-$ $n)\left.\right|^{2}$ is bounded on $[0,1]$ and since $\sum_{m=0}^{N-1}|\psi(y-m / N)|^{2}$ is bounded on $[0,1 / N]$, it is easy to deduce that any function $f$ in $\mathscr{A}_{\varphi, \psi}$ is a continuous function defined by the pointwise sum

$$
f(x, y)=\sum_{n \in \mathbb{Z}} \sum_{m=0}^{N-1} a_{n, m} \varphi(x-n) \psi\left(y-\frac{m}{N}\right)
$$

in $\mathbb{R} \times[0,1)$.

Besides, the subspace $\mathscr{A}_{\varphi, \psi}$ is a reproducing kernel Hilbert space (RKHS) since the evaluation functionals are bounded in $\mathscr{A}_{\varphi, \psi}$. Namely, using Cauchy-Schwarz's inequality in (44) and Riesz basis definition, for each $(x, y) \in \mathbb{R} \times[0,1)$, we have

$$
\begin{array}{r}
|f(x, y)|^{2} \leq \frac{\|f\|^{2}}{A} \sum_{n \in \mathbb{Z}}|\varphi(x-n)|^{2} \sum_{m=0}^{N-1}\left|\psi\left(y-\frac{m}{N}\right)\right|^{2}, \\
f \in \mathscr{A}_{\varphi, \psi},
\end{array}
$$

where $A$ denotes the lower Riesz bound of the Riesz basis $\{\varphi(x-n) \psi(y-m / N)\}_{n, m}$ for $\mathscr{A}_{\varphi, \psi}$. The above inequality shows that convergence in $L^{2}(\mathbb{R} \times(0,1))$ implies pointwise convergence which is uniform on $\mathbb{R} \times[0,1)$. See, for instance, [31] for the relationship between sampling theory and RKHS.

\section{Infinite-Infinite Generators Case}

Let $\mathscr{H}_{1}$ and $\mathscr{H}_{2}$ be two separable Hilbert spaces and $U$ : $\mathscr{H}_{1} \rightarrow \mathscr{H}_{1}$ and $V: \mathscr{H}_{2} \rightarrow \mathscr{H}_{2}$ two unitary operators. Consider two elements $a \in \mathscr{H}_{1}$ and $b \in \mathscr{H}_{2}$ such that the sequences $\left\{U^{n} a\right\}_{n \in \mathbb{Z}}$ and $\left\{V^{m} b\right\}_{m \in \mathbb{Z}}$ form a Riesz sequence in $\mathscr{H}_{1}$ and in $\mathscr{H}_{2}$, respectively. In the tensor product Hilbert space $\mathscr{H}_{1} \otimes \mathscr{H}_{2}$ we consider its closed subspace

$$
\mathscr{A}_{a, b}:=\overline{\operatorname{span}}_{\mathscr{H}_{1} \otimes \mathscr{H}_{2}}\left\{U^{n} a \otimes V^{m} b\right\}_{n, m \in \mathbb{Z}} \cdot
$$

Since the sequence $\left\{U^{n} a \otimes V^{m} b\right\}_{n, m \in \mathbb{Z}}$ is a Riesz basis for the tensor product $\mathscr{A}_{a} \otimes \mathscr{A}_{b}$ of the $U$-invariant subspace $\mathscr{A}_{a}=$ $\left\{\sum_{n \in \mathbb{Z}} a_{n} U^{n} a:\left\{a_{n}\right\} \in \ell^{2}(\mathbb{Z})\right\}$ in $\mathscr{H}_{1}$ and the $V$-invariant subspace $\mathscr{A}_{b}=\left\{\sum_{m \in \mathbb{Z}} b_{m} V^{m} b:\left\{b_{m}\right\} \in \ell^{2}(\mathbb{Z})\right\}$ in $\mathscr{H}_{2}$ we deduce that $\mathscr{A}_{a, b}=\mathscr{A}_{a} \otimes \mathscr{A}_{b}$ which can be described as

$$
\mathscr{A}_{a, b}=\left\{\sum_{n, m \in \mathbb{Z}} a_{n m} U^{n} a \otimes V^{m} b:\left\{a_{n m}\right\} \in \ell^{2}\left(\mathbb{Z}^{2}\right)\right\} .
$$

We will refer to the vectors $\{a, b\}$ as the infinite-infinite generators of the subspace $\mathscr{A}_{a, b}$ in $\mathscr{H}_{1} \otimes \mathscr{H}_{2}$.

The Samples. For fixed $S=s s^{\prime}$ elements $h_{j j^{\prime}}:=h_{j 1} \otimes h_{j^{\prime} 2} \in$ $\mathscr{H}_{1} \otimes \mathscr{H}_{2}, j=1,2, \ldots, s, j^{\prime}=1,2, \ldots, s^{\prime}$, we consider two sampling periods $r, \bar{r} \in \mathbb{N}$. For each $x \in \mathscr{A}_{a, b}$ we introduce the sequence of its generalized samples

$$
\left\{\mathscr{L}_{j j^{\prime}} x(r n, \bar{r} m)\right\}_{n \in \mathbb{Z} ; j=1,2, \ldots, s ; m \in \mathbb{Z} ; j^{\prime}=1,2, \ldots, s^{\prime}}
$$

defined by

$$
\mathscr{L}_{j j^{\prime}} x(r n, \bar{r} m):=\left\langle x, U^{r n} h_{j 1} \otimes V^{\bar{r} m} h_{j^{\prime} 2}\right\rangle_{\mathscr{H}_{1} \otimes \mathscr{H}_{2}},
$$

where $n, m \in \mathbb{Z}, j=1,2, \ldots, s$, and $j^{\prime}=1,2, \ldots, s^{\prime}$.

The Isomorphism $\mathscr{T}_{a, b}^{U V}$. In this case we introduce the isomorphism $\mathscr{T}_{a, b}^{U V}$ which maps the orthonormal basis $\left\{e^{2 \pi i n x} \otimes\right.$ $\left.e^{2 \pi i m x}\right\}_{n, m \in \mathbb{Z}}$ for the Hilbert space $L^{2}(0,1) \otimes L^{2}(0,1)$ onto the Riesz basis $\left\{U^{n} a \otimes V^{m} b\right\}_{n, m \in \mathbb{Z}}$ for $\mathscr{A}_{a, b}$. That is, $\mathscr{T}_{a, b}^{U V}=\mathscr{T}_{a}^{U} \otimes$ $\mathscr{T}_{b}^{V}$, where $\mathscr{T}_{a}^{U}$ and $\mathscr{T}_{b}^{V}$ denote the isomorphisms

$$
\begin{aligned}
\mathscr{T}_{a}^{U}: & L^{2}(0,1) \longrightarrow \mathscr{A}_{a} \\
e^{2 \pi i n x} & \longmapsto U^{n} a, \\
\mathscr{T}_{b}^{V}: \quad L^{2}(0,1) & \longrightarrow \mathscr{A}_{b} \\
& e^{2 \pi i m x} \longmapsto V^{m} b .
\end{aligned}
$$

Here, the shifting property reads as follows:

$$
\begin{gathered}
\mathscr{T}_{a, b}^{U V}\left(g(x) e^{2 \pi i M x} \otimes \widetilde{g}(x) e^{2 \pi i N x}\right) \\
=U^{M}\left(\mathscr{T}_{a}^{U} g\right) \otimes V^{N}\left(\mathscr{T}_{b}^{V} \tilde{g}\right) \\
=\left(U^{M} \otimes V^{N}\right) \mathscr{T}_{a, b}^{U V}(g \otimes \tilde{g}),
\end{gathered}
$$


where $g, \tilde{g} \in L^{2}(0,1)$ and $N, M \in \mathbb{Z}$. The proof of (51) goes in the same manner as in Lemma 1.

An Expression for the Samples. For any $x=\sum_{k, p \in \mathbb{Z}} a_{k p} U^{k} a \otimes$ $V^{p} b$ in $\mathscr{A}_{a, b}$ we have

$$
\begin{aligned}
& \mathscr{L}_{j j^{\prime}} x(r n, \bar{r} m) \\
& =\left\langle\sum_{k, p \in \mathbb{Z}} a_{k p} U^{k} a \otimes V^{p} b, U^{r n} h_{j 1} \otimes V^{\bar{r} m} h_{j^{\prime} 2}\right\rangle_{\mathscr{H}_{1} \otimes \mathscr{H}_{2}} \\
& =\sum_{k, p \in \mathbb{Z}} a_{k p}\left\langle U^{k} a \otimes V^{p} b, U^{r n} h_{j 1} \otimes V^{\bar{r} m} h_{j^{\prime} 2}\right\rangle_{\mathscr{H}_{1} \otimes \mathscr{H}_{2}} .
\end{aligned}
$$

Now, consider $F:=\sum_{k, p \in \mathbb{Z}} a_{k p} e^{2 \pi i k x} \otimes e^{2 \pi i p x}$, which is the element in $L^{2}(0,1) \otimes L^{2}(0,1)$ such that $\mathscr{T}_{a, b}^{U V} F=x$. Thus, Plancherel identity for orthonormal bases gives

$$
\begin{aligned}
& \mathscr{L}_{j j^{\prime}} x(r n, \bar{r} m)=\langle F, \\
& \sum_{k, p \in \mathbb{Z}}\left\langle\overline{a, U^{r n-k} h_{j 1}}\right\rangle_{\mathscr{H}_{1}}\left\langle\overline{b, V^{\bar{r} m-p} h_{j^{\prime} 2}}\right\rangle_{\mathscr{H}_{2}} e^{2 \pi i k x} \\
& \left.\otimes e^{2 \pi i p x}\right\rangle_{L^{2}(0,1) \otimes L^{2}(0,1)}=\langle F, \\
& \left(\sum_{k \in \mathbb{Z}}\left\langle\overline{a, U^{r n-k} h_{j 1}}\right\rangle_{\mathscr{H}_{1}} e^{2 \pi i k x}\right) \\
& \left.\otimes\left(\sum_{p \in \mathbb{Z}}\left\langle\overline{b, V^{\bar{r} m-p} h_{j^{\prime} 2}}\right\rangle_{\mathscr{H}_{2}} e^{2 \pi i p x}\right)\right\rangle_{L^{2}(0,1) \otimes L^{2}(0,1)}
\end{aligned}
$$

That is, for $n, m \in \mathbb{Z}, j=1,2, \ldots, s$, and $j^{\prime}=1,2, \ldots, s^{\prime}$ we have got the following expression for the samples:

$$
\begin{aligned}
& \mathscr{L}_{j j^{\prime}} x(r n, \bar{r} m) \\
& \quad=\left\langle F, \overline{g_{j 1}(x)} e^{2 \pi i r n x} \otimes \overline{g_{j^{\prime} 2}(x)} e^{2 \pi i \bar{r} m x}\right\rangle_{L^{2}(0,1) \otimes L^{2}(0,1)},
\end{aligned}
$$

where, for $j=1,2, \ldots, s$ and $j^{\prime}=1,2, \ldots, s^{\prime}$, the functions

$$
\begin{aligned}
g_{j 1}(x) & :=\sum_{k \in \mathbb{Z}}\left\langle a, U^{k} h_{j 1}\right\rangle_{\mathscr{H}_{1}} e^{2 \pi i k x}, \\
g_{j^{\prime} 2}(x) & :=\sum_{k \in \mathbb{Z}}\left\langle b, V^{k} h_{j^{\prime} 2}\right\rangle_{\mathscr{H}_{2}} e^{2 \pi i k x}
\end{aligned}
$$

belong to $L^{2}(0,1)$. Thus, we deduce the following result.

Proposition 8. Any element $x \in \mathscr{A}_{a, b}$ can be recovered from the sequence of its samples $\left\{\mathscr{L}_{j j^{\prime}} x(\mathrm{rn}\right.$, $\bar{r} m)\}_{n \in \mathbb{Z} ; j=1,2, \ldots, s ; m \in \mathbb{Z} ; j^{\prime}=1,2, \ldots, s^{\prime}}$ in a stable way (by means of a frame expansion) if and only if the sequence $\left\{\overline{g_{j 1}(x)} e^{2 \pi i r n x} \otimes \overline{g_{j^{\prime} 2}(x)} e^{2 \pi i r m x}\right\}_{n \in \mathbb{Z} ; j=1,2, \ldots, s ; m \in \mathbb{Z} ; j^{\prime}=1,2, \ldots, s^{\prime}}$ is a frame for $L^{2}(0,1) \otimes L^{2}(0,1)$.

The thesis of the above proposition is true if and only if $0<\alpha_{\mathbf{G}_{1}} \leq \beta_{\mathbf{G}_{1}}<\infty$ and $0<\alpha_{\mathbf{G}_{2}} \leq \beta_{\mathbf{G}_{2}}<\infty$, where $\mathbf{G}_{1}$ and $\mathbf{G}_{2}$ denote the $s \times r$ and $s^{\prime} \times \bar{r}$ matrices defined in (22) for $g_{j 1}$, $j=1,2, \ldots, s$, and $g_{j^{\prime}}, j^{\prime}=1,2, \ldots, s^{\prime}$, respectively.

4.1. The Sampling Result. In case that $\left\{\overline{g_{j 1}(x)} e^{2 \pi i r n x} \otimes\right.$ $\left.\overline{g_{j^{\prime} 2}(x)} e^{2 \pi i \bar{r} m x}\right\}_{n \in \mathbb{Z} ; j=1,2, \ldots, s ; m \in \mathbb{Z} ; j^{\prime}=1,2, \ldots, s^{\prime}}$ is a frame for $L^{2}(0,1) \otimes$ $L^{2}(0,1)$, a family of dual frames is given by $\left\{r h_{j 1}(x) e^{2 \pi i r n x} \otimes\right.$ $\left.\bar{r} h_{j^{\prime} 2}(x) e^{2 \pi i \bar{r} m x}\right\}_{n \in \mathbb{Z} ; j=1,2, \ldots, s ; m \in \mathbb{Z} ; j^{\prime}=1,2, \ldots, s^{\prime}}$, where the functions $h_{j 1}, j=1,2, \ldots, s$, and $h_{j^{\prime} 2}, j^{\prime}=1,2, \ldots, s^{\prime}$, in $L^{\infty}(0,1)$, satisfy

$$
\begin{aligned}
& {\left[h_{11}(x), h_{21}(x), \ldots, h_{s 1}(x)\right] \mathbf{G}_{1}(x)=[1,0, \ldots, 0],} \\
& {\left[h_{12}(x), h_{22}(x), \ldots, h_{s^{\prime} 2}(x)\right] \mathbf{G}_{2}(x)=[1,0, \ldots, 0]} \\
& \text { a.e. in }(0,1) \text {. }
\end{aligned}
$$

Thus, for each $x \in \mathscr{A}_{a, b}$ there exists a unique $F \in L^{2}(0,1) \otimes$ $L^{2}(0,1)$ such that $\mathscr{T}_{a, b}^{U V} F=x$. This $F$ can be expressed in $L^{2}(0,1) \otimes L^{2}(0,1)$ as the frame expansion

$$
F=\sum_{j=1}^{s} \sum_{j^{\prime}=1}^{s^{\prime}} \sum_{n \in \mathbb{Z}} \sum_{m \in \mathbb{Z}} \mathscr{L}_{j j^{\prime}} x(r n, \bar{r} m)\left(r h_{j 1}(x) e^{2 \pi i r n x} \otimes \bar{r} h_{j^{\prime} 2}(x) e^{2 \pi i \bar{r} m x}\right) .
$$

Then, applying the isomorphism $\mathscr{T}_{a, b}^{U V}$ and (51) one gets

$$
\begin{aligned}
x & =\sum_{j=1}^{s} \sum_{j^{\prime}=1}^{s^{\prime}} \sum_{n \in \mathbb{Z}} \sum_{m \in \mathbb{Z}} \mathscr{L}_{j j^{\prime}} x(r n, \bar{r} m) \mathscr{T}_{a, b}^{U V}\left(r h_{j 1}(x) e^{2 \pi i r n x} \otimes \bar{r}_{j^{\prime} 2}(x) e^{2 \pi i \bar{r} m x}\right) \\
& =\sum_{j=1}^{s} \sum_{j^{\prime}=1}^{s^{\prime}} \sum_{n \in \mathbb{Z}} \sum_{m \in \mathbb{Z}} \mathscr{L}_{j j^{\prime}} x(r n, \bar{r} m) U^{r n}\left(\mathscr{T}_{a}^{U} r h_{j 1}\right) \otimes V^{\bar{r} m}\left(\mathscr{T}_{b}^{V} \bar{r} h_{j^{\prime} 2}\right) \\
& =\sum_{j=1}^{s} \sum_{j^{\prime}=1}^{s^{\prime}} \sum_{n \in \mathbb{Z}} \sum_{m \in \mathbb{Z}} \mathscr{L}_{j j^{\prime}} x(r n, \bar{r} m)\left(U^{r n} \otimes V^{\bar{r} m}\right)\left(c_{j 1} \otimes d_{j^{\prime} 2}\right),
\end{aligned}
$$


where $c_{j 1}=\mathscr{T}_{a}^{U}\left(r h_{j 1}\right) \in \mathscr{A}_{a}, j=1,2, \ldots, s$, and $d_{j^{\prime} 2}=$ $\mathscr{T}_{b}^{V}\left(\bar{r} h_{j^{\prime} 2}\right) \in \mathscr{A}_{b}, j^{\prime}=1,2, \ldots, s^{\prime}$. Next, we state the equivalent result to Theorem 6; its proof goes in the same manner.

Theorem 9. Let $h_{j j^{\prime}}:=h_{j 1} \otimes h_{j^{\prime} 2} \in \mathscr{H}_{1} \otimes \mathscr{H}_{2}, j=1,2, \ldots, s$, and $j^{\prime}=1,2, \ldots, s^{\prime}$. Let $\mathscr{L}_{j j^{\prime}}$ be the associated $U V$-system giving the samples of any $x \in \mathscr{A}_{a, b}$ as in (49), $j=1,2, \ldots$, s and $j^{\prime}=1,2, \ldots, s^{\prime}$. Assume that the functions $g_{j 1}$ and $g_{j^{\prime} 2}, j=1$, $2, \ldots$, s and $j^{\prime}=1,2, \ldots, s^{\prime}$, given in (55) belong to $L^{\infty}(0,1)$ or, equivalently, that $\beta_{\mathbf{G}_{1}}<\infty$ and $\beta_{\mathbf{G}_{2}}<\infty$ for the correspondent $s \times r$ and $s^{\prime} \times \bar{r}$ matrices $\mathbf{G}_{1}(x)$ and $\mathbf{G}_{2}(x)$, respectively, defined in (22). The following statements are equivalent:

(a) $\alpha_{\mathbf{G}_{1}}>0$ and $\alpha_{\mathbf{G}_{2}}>0$.

(b) There exist $S=s^{\prime}$ elements $c_{j} \otimes d_{j^{\prime}}$ in the subspace $\mathscr{A}_{a, b}, j=1,2, \ldots, s$ and $j^{\prime}=1,2, \ldots, s^{\prime}$, such that the sequence $\left\{\left(U^{r n} \otimes V^{\bar{r} m}\right)\left(c_{j} \otimes d_{j^{\prime}}\right)\right\}_{n \in \mathbb{Z} ; j=1,2, \ldots, s ; m \in \mathbb{Z} ; j^{\prime}=1,2, \ldots, s^{\prime}}$ is a frame for $\mathscr{A}_{a, b}$, and for any $x \in \mathscr{A}_{a, b}$ the expansion

$x$

$$
\begin{array}{r}
=\sum_{j=1}^{s} \sum_{j^{\prime}=1}^{s^{\prime}} \sum_{n \in \mathbb{Z}} \sum_{m \in \mathbb{Z}} \mathscr{L}_{j j^{\prime}} x(r n, \bar{r} m)\left(U^{r n} \otimes V^{\bar{r} m}\right)\left(c_{j} \otimes d_{j^{\prime}}\right) \\
\text { in } \mathscr{H}_{1} \otimes \mathscr{H}_{2}
\end{array}
$$

holds.

(c) There exists a frame $\left\{C_{j, j^{\prime}, n, m}\right\}_{n \in \mathbb{Z} ; j=1,2, \ldots, s ; m \in \mathbb{Z} ; j^{\prime}=1,2, \ldots, s^{\prime}}$ for $\mathscr{A}_{a, b}$ such that, for each $x \in \mathscr{A}_{a, b}$, the expansion

$$
x=\sum_{j=1}^{s} \sum_{j^{\prime}=1}^{s^{\prime}} \sum_{n \in \mathbb{Z}} \sum_{m \in \mathbb{Z}} \mathscr{L}_{j j^{\prime}} x(r n, \bar{r} m) C_{j, j^{\prime}, n, m}
$$

$$
\text { in } \mathscr{H}_{1} \otimes \mathscr{H}_{2}
$$

holds.

In cases $s=r$ and $s^{\prime}=\bar{r}$ we are in the Riesz bases setting and the theorem above admits the following corollary.

Corollary 10. In addition to the hypotheses of theorem above, assume that $s=r$ and $s^{\prime}=\bar{r}$. The following statements are equivalent:

(1) $\alpha_{\mathbf{G}_{1}}>0$ and $\alpha_{\mathbf{G}_{2}}>0$.

(2) There exist $r \bar{r}$ unique elements $c_{j} \otimes d_{j^{\prime}}$ in the subspace $\mathscr{A}_{a, b}, j=1,2, \ldots, r$ and $j^{\prime}=1,2, \ldots, \bar{r}$, such that the sequence $\left\{\left(U^{r n} \otimes V^{r m}\right)\left(c_{j} \otimes d_{j^{\prime}}\right)\right\}_{n \in \mathbb{Z} ; j=1,2, \ldots, r ; m \in \mathbb{Z} ; j^{\prime}=1,2, \ldots, \bar{r}}$ is a Riesz basis for $\mathscr{A}_{a, b}$, and the expansion of any $x \in$ $\mathscr{A}_{a, b}$ with respect to this basis is

$x$

$$
=\sum_{j=1}^{r} \sum_{j^{\prime}=1}^{\bar{r}} \sum_{n \in \mathbb{Z}} \sum_{m \in \mathbb{Z}} \mathscr{L}_{j j^{\prime}} x(r n, \bar{r} m)\left(U^{r n} \otimes V^{r m}\right)\left(c_{j} \otimes d_{j^{\prime}}\right)
$$

$$
\text { in } \mathscr{H}_{1} \otimes \mathscr{H}_{2} \text {. }
$$

In case that the equivalent conditions are satisfied, the vectors $c_{j} \otimes d_{j^{\prime}}, j=1,2, \ldots, r$ and $j^{\prime}=1,2, \ldots, \bar{r}$, satisfy the interpolation property

$$
\mathscr{L}_{k k^{\prime}}\left(c_{j} \otimes d_{j^{\prime}}\right)(r n, r m)=\delta_{j, k} \delta_{j^{\prime}, k^{\prime}} \delta_{n, 0} \delta_{m, 0},
$$

whenever $n, m \in \mathbb{Z}, j, k=1,2, \ldots, r$, and $j^{\prime}, k^{\prime}=1,2, \ldots, \bar{r}$.

A Representative Example. In $\mathscr{H}_{1}=\mathscr{H}_{2}:=L^{2}(\mathbb{R})$ consider $U=V$ the shift operator $f(x) \mapsto f(x-1)$. Let $\varphi, \psi \in$ $L^{2}(\mathbb{R})$ be two functions such that the sequences $\{\varphi(x-n)\}_{n \in \mathbb{Z}}$ and $\{\psi(y-m)\}_{m \in \mathbb{Z}}$ are Riesz sequences for $L^{2}(\mathbb{R})$ (e.g., the functions $\varphi$ and $\psi$ may be two $B$-splines).

In the tensor product $L^{2}(\mathbb{R}) \otimes L^{2}(\mathbb{R})=L^{2}\left(\mathbb{R}^{2}\right)$ consider the closed subspace $\mathscr{A}_{\varphi, \psi}:=\mathscr{A}_{\varphi} \otimes \mathscr{A}_{\psi}$, that is, the tensor product of the shift-invariant subspaces $\mathscr{A}_{\varphi}$ and $\mathscr{A}_{\psi}$ of $L^{2}(\mathbb{R})$. Given the functions $h_{j 1}, h_{j^{\prime} 2} \in L^{2}(\mathbb{R}), j=1,2, \ldots, s$ and $j^{\prime}=1,2, \ldots, s^{\prime}$, and fixing the sampling periods $r$ and $\bar{r}$ in $\mathbb{N}$, for each $f$ in $\mathscr{A}_{\varphi, \psi}$ we consider its samples defined by

$$
\begin{aligned}
& \mathscr{L}_{j j^{\prime}} f(r n, \bar{r} m) \\
& \quad:=\iint_{-\infty}^{\infty} f(x, y) \overline{h_{j 1}(x-r n)} \overline{h_{j^{\prime} 2}(y-\bar{r} m)} d x d y,
\end{aligned}
$$

$n, m \in \mathbb{Z}$

Under the hypotheses in Theorem 9 there will exist functions $S_{j} \in \mathscr{A}_{\varphi}, j=1,2, \ldots, s$, and $\widetilde{S}_{j^{\prime}} \in \mathscr{A}_{\psi}, j^{\prime}=1,2, \ldots, s^{\prime}$, such that for any $f \in \mathscr{A}_{\varphi, \psi}$ the sampling expansion (59) reads as follows:

$$
\begin{array}{r}
f(x, y) \\
=\sum_{j=1}^{s} \sum_{j^{\prime}=1}^{s^{\prime}} \sum_{n \in \mathbb{Z}} \sum_{m \in \mathbb{Z}} \mathscr{L}_{j j^{\prime}} f(r n, \bar{r} m) S_{j}(x-r n) \widetilde{S}_{j^{\prime}}(y-\bar{r} m) \\
\text { in } L^{2}\left(\mathbb{R}^{2}\right) .
\end{array}
$$

As in the example of the infinite-finite case, assuming that the generators $\varphi$ and $\psi$ are continuous functions on $\mathbb{R}$ such that the sums $\sum_{n \in \mathbb{Z}}|\varphi(x-n)|^{2}$ and $\sum_{m \in \mathbb{Z}}|\psi(y-m)|^{2}$ are bounded on $[0,1]$, it is easy to deduce that $\mathscr{A}_{\varphi, \psi}$ is a RKHS of continuous functions on $\mathbb{R}^{2}$. Furthermore, the convergence in $L^{2}\left(\mathbb{R}^{2}\right)$ implies pointwise convergence which is uniform on $\mathbb{R}^{2}$.

\section{Finite-Finite Generators Case}

Let $\mathscr{H}_{1}$ and $\mathscr{H}_{2}$ be two separable Hilbert spaces and $U$ : $\mathscr{H}_{1} \rightarrow \mathscr{H}_{1}$ and $V: \mathscr{H}_{2} \rightarrow \mathscr{H}_{2}$ two unitary operators. Consider two elements $a \in \mathscr{H}_{1}$ and $b \in \mathscr{H}_{2}$ such that, for some $N, M \in \mathbb{N}, U^{N} a=a$ and $V^{M} b=b$ and the sets $\left\{a, U a, U^{2} a, \ldots, U^{N-1} b\right\}$ and $\left\{b, V b, V^{2} b, \ldots, V^{M-1} b\right\}$ are linearly independent in $\mathscr{H}_{1}$ and $\mathscr{H}_{2}$, respectively. In the 
tensor product Hilbert space $\mathscr{H}_{1} \otimes \mathscr{H}_{2}$ we consider the finite subspace

$$
\begin{aligned}
\mathscr{A}_{a, b} & :=\operatorname{span}\left\{U^{p} a \otimes V^{q} b\right\}_{p=0,1, \ldots, N-1 ; q=0,1, \ldots, M-1} \\
& =\left\{\sum_{p=0}^{N-1} \sum_{q=0}^{M-1} a_{p q} U^{p} a \otimes V^{q} b\right\} .
\end{aligned}
$$

The subspace $\mathscr{A}_{a, b}$ coincides with the tensor product $\mathscr{A}_{a} \otimes \mathscr{A}_{b}$ of the finite subspaces $\mathscr{A}_{a}=\left\{\sum_{p=0}^{N-1} a_{p} U^{p} a: a_{p} \in \mathbb{C}\right\} \subset \mathscr{H}_{1}$ and $\mathscr{A}_{b}=\left\{\sum_{q=0}^{M-1} b_{q} V^{q} b: b_{q} \in \mathbb{C}\right\} \subset \mathscr{H}_{2}$. We will refer to the vectors $\{a, b\}$ as the finite-finite generators of the subspace $\mathscr{A}_{a, b}$ in $\mathscr{H}_{1} \otimes \mathscr{H}_{2}$.

The Samples. For fixed $S=s s^{\prime}$ elements $h_{j j^{\prime}}:=h_{j 1} \otimes h_{j^{\prime} 2} \in$ $\mathscr{H}_{1} \otimes \mathscr{H}_{2}, j=1,2, \ldots, s$ and $j^{\prime}=1,2, \ldots, s^{\prime}$, we consider two sampling periods, $r$ a divisor of $N$ and $\bar{r}$ a divisor of $M$; denote $\ell:=N / r$ and $\bar{\ell}:=M / \bar{r}$. For each $x \in \mathscr{A}_{a, b}$ we introduce its Se $\bar{\ell}$ generalized samples

$$
\left\{\mathscr{L}_{j j^{\prime}} x(r n, \bar{r} m)\right\}_{n=0,1, \ldots, \ell-1 ; j=1,2, \ldots, s ; m=0,1, \ldots, \bar{\ell}-1 ; j^{\prime}=1,2, \ldots, s^{\prime}}
$$

defined by

$$
\mathscr{L}_{j j^{\prime}} x(r n, \bar{r} m):=\left\langle x, U^{r n} h_{j 1} \otimes V^{\bar{r} m} h_{j^{\prime} 2}\right\rangle_{\mathscr{H}_{1} \otimes \mathscr{H}_{2}},
$$

where $n=0,1, \ldots, \ell-1, m=0,1, \ldots, \bar{\ell}-1, j=1,2, \ldots, s$, and $j^{\prime}=1,2, \ldots, s^{\prime}$.

The Isomorphism $\mathscr{T}_{a, b}^{U V}$. As in the former cases, now we introduce the isomorphism $\mathscr{T}_{a, b}^{U V}$ which maps the orthonormal basis $\left\{\mathbf{e}_{p} \otimes \widetilde{\mathbf{e}}_{q}\right\}_{p=0,1, \ldots, N-1 ; q=0,1, \ldots, M-1}$ for the Hilbert space $\ell_{N}^{2}(\mathbb{Z}) \otimes \ell_{M}^{2}(\mathbb{Z})$ onto the basis $\left\{U^{p} a \otimes V^{q} b\right\}_{p=0,1, \ldots, N-1 ; q=0,1, \ldots, M-1}$ for $\mathscr{A}_{a, b}$; here $\left\{\mathbf{e}_{p}\right\}_{p=0}^{N-1}$ and $\left\{\widetilde{\mathbf{e}}_{q}\right\}_{q=0}^{M-1}$ denote, respectively, the canonical bases for $\ell_{N}^{2}(\mathbb{Z})$ and $\ell_{M}^{2}(\mathbb{Z})$. In other words, $\mathscr{T}_{a, b}^{U V}=$ $\mathscr{T}_{a, N}^{U} \otimes \mathscr{T}_{b, M}^{V}$, where $\mathscr{T}_{a, N}^{U}$ and $\mathscr{T}_{b, M}^{V}$ denote the isomorphisms

$$
\begin{aligned}
\mathscr{T}_{a, N}^{U}: & e_{N}^{2}(\mathbb{Z}) \longrightarrow \mathscr{A}_{a} \\
& \mathbf{e}_{p} \longmapsto U^{p} a, \\
\mathscr{T}_{b, M}^{V}: & \ell_{M}^{2}(\mathbb{Z}) \longrightarrow \mathscr{A}_{b} \\
& \widetilde{\mathbf{e}}_{q} \longmapsto V^{q} b .
\end{aligned}
$$

Here, the shifting property reads as follows:

$$
\begin{aligned}
\mathscr{T}_{a, b}^{U V}\left(\mathbb{\mathbb { T }}_{N-p} \otimes \widetilde{\mathbb{T}}_{M-q}\right)= & U^{p}\left(\mathscr{T}_{a, N}^{U} \mathbb{\mathbb { T }}_{0}\right) \\
& \otimes V^{q}\left(\mathscr{T}_{b, M}^{V} \widetilde{\mathbb{T}}_{0}\right) \\
= & \left(U^{p} \otimes V^{q}\right) \mathscr{T}_{a, b}^{U V}\left(\mathbb{\mathbb { T }}_{0} \otimes \widetilde{\mathbb{T}}_{0}\right),
\end{aligned}
$$

where

$$
\begin{gathered}
\mathbb{T}_{0}=(T(0), T(1), \ldots, T(N-1)), \\
\mathbb{\mathbb { V }}_{N-p}=(T(N-p), T(N-p+1), \ldots, \\
T(N-p+N-1))
\end{gathered}
$$

belong to $\ell_{N}^{2}(\mathbb{Z})$ and $1 \leq p \leq N-1$, and

$$
\begin{aligned}
& \widetilde{\mathbb{T}}_{0}=(\widetilde{T}(0), \widetilde{T}(1), \ldots, \widetilde{T}(M-1)), \\
& \widetilde{\mathbb{T}}_{M-q}=(\widetilde{T}(M-q), \widetilde{T}(M-q+1), \ldots, \\
& \tilde{T}(M-q+M-1))
\end{aligned}
$$

belong to $\ell_{M}^{2}(\mathbb{Z})$ and $1 \leq q \leq M-1$. The proof of (69) goes in the same manner as in Lemma 1.

An Expression for the Samples. For any $x=$ $\sum_{p=0}^{N-1} \sum_{q=0}^{M-1} a_{p q} U^{p} a \otimes V^{q} b$ in $\mathscr{A}_{a, b}$ we have

$$
\begin{aligned}
& \mathscr{L}_{j j^{\prime}} x(r n, \bar{r} m)=\left\langle\sum_{p=0}^{N-1} \sum_{q=0}^{M-1} a_{p q} U^{p} a \otimes V^{q} b, U^{r n} h_{j 1}\right. \\
& \left.\otimes V^{\bar{r} m} h_{j^{\prime} 2}\right\rangle_{\mathscr{H}_{1} \otimes \mathscr{H}_{2}}=\sum_{p=0}^{N-1} \sum_{q=0}^{M-1} a_{p q}\left\langle U^{p} a\right. \\
& \left.\otimes V^{q} b, U^{r n} h_{j 1} \otimes V^{\bar{r} m} h_{j^{\prime} 2}\right\rangle_{\mathscr{H}_{1} \otimes \mathscr{H}_{2}} .
\end{aligned}
$$

Now, consider $F:=\sum_{p=0}^{N-1} \sum_{q=0}^{M-1} a_{p q} \mathbf{e}_{p} \otimes \widetilde{\mathbf{e}}_{q}$, which is the element in $\ell_{N}^{2}(\mathbb{Z}) \otimes \ell_{M}^{2}(\mathbb{Z})$ such that $\mathscr{T}_{a, b}^{U V} F=x$. Thus, Plancherel identity for orthonormal bases gives

$$
\begin{aligned}
& \mathscr{L}_{j j^{\prime}} x(r n, \bar{r} m)=\langle F, \\
& \sum_{p=0}^{N-1} \sum_{q=0}^{M-1}\left\langle\overline{a, U^{r n-p} h_{j 1}}\right\rangle_{\mathscr{H}_{1}}\left\langle\overline{b, V^{\bar{r} m-q} h_{j^{\prime} 2}}\right\rangle_{\mathscr{H}_{2}} \mathbf{e}_{p} \\
& \left.\otimes \widetilde{\mathbf{e}}_{q}\right\rangle_{\ell_{N}^{2}(\mathbb{Z}) \otimes \ell_{M}^{2}(\mathbb{Z})}=\langle F, \\
& \left(\sum_{p=0}^{N-1}\left\langle\overline{a, U^{r n-p} h_{j 1}}\right\rangle_{\mathscr{H}_{1}} \mathbf{e}_{p}\right) \\
& \left.\otimes\left(\sum_{q=0}^{M-1}\left\langle\overline{b, V^{\bar{r} m-q h_{j^{\prime} 2}}}\right\rangle_{\mathscr{H}_{2}} \widetilde{\mathbf{e}}_{q}\right)\right\rangle_{\ell_{N}^{2}(\mathbb{Z}) \otimes \ell_{M}^{2}(\mathbb{Z})} .
\end{aligned}
$$

That is, for $n=0,1, \ldots, \ell-1, m=0,1, \ldots, \bar{\ell}-1, j=1,2, \ldots, s$, and $j^{\prime}=1,2, \ldots, s^{\prime}$ we have got the following expression for the samples:

$$
\mathscr{L}_{j j^{\prime}} x(r n, \bar{r} m)=\left\langle F, \mathbb{G}_{j, n}^{1} \otimes \mathbb{G}_{j^{\prime}, m}^{2}\right\rangle_{\ell_{N}^{2}(\mathbb{Z}) \otimes \ell_{M}^{2}(\mathbb{Z})},
$$

where

$$
\begin{aligned}
\mathbb{G}_{j, n}^{1} & :=\sum_{p=0}^{N-1}\left\langle\overline{\left\langle, U^{r n-p} h_{j 1}\right.}\right\rangle_{\mathscr{H}_{1}} \mathbf{e}_{p} \\
& =\sum_{p=0}^{N-1} \overline{R_{a, h_{j 1}}(N+p-r m)} \mathbf{e}_{p},
\end{aligned}
$$




$$
\begin{aligned}
\mathbb{G}_{j^{\prime}, m}^{2} & :=\sum_{q=0}^{M-1}\left\langle\overline{b, V^{\bar{r} m-q} h_{j^{\prime} 2}}\right\rangle_{\mathscr{H}_{2}} \widetilde{\mathbf{e}}_{q} \\
& =\sum_{q=0}^{M-1} \overline{R_{b, h_{j^{\prime} 2}}(M+q-\bar{r} m)} \widetilde{\mathbf{e}}_{q} .
\end{aligned}
$$

Here, $R_{a, h_{j 1}}(k):=\left\langle U^{k} a, h_{j 1}\right\rangle_{\mathscr{H}_{1}}, k \in \mathbb{Z}$, denotes the $(N$ periodic) cross-covariance sequence between the sequences $\left\{U^{k} a\right\}_{k \in \mathbb{Z}}$ and $\left\{U^{k} h_{j 1}\right\}_{k \in \mathbb{Z}}$ in $\mathscr{H}_{1}$. Similarly, $R_{b, h_{j^{\prime} 2}}(k):=$ $\left\langle V^{k} b, h_{j^{\prime} 2}\right\rangle_{\mathscr{C}_{2}}, k \in \mathbb{Z}$, denotes the (M-periodic) crosscovariance sequence between the sequences $\left\{V^{k} b\right\}_{k \in \mathbb{Z}}$ and $\left\{V^{k} h_{j^{\prime} 2}\right\}_{k \in \mathbb{Z}}$ in $\mathscr{H}_{2}$. Thus, we deduce the following result.

Proposition 11. Any element $x \in \mathscr{A}_{a, b}$ can be recovered from the sequence of its samples $\left\{\mathscr{L}_{j j^{\prime}} x(\mathrm{rn}\right.$,

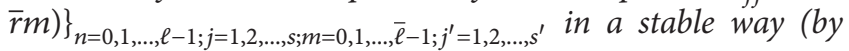
means of a frame expansion) if and only if the sequence $\left\{\mathbb{G}_{j, n}^{1} \otimes \mathbb{G}_{j^{\prime}, m}^{2}\right\}_{n=0,1, \ldots, \ell-1 ; j=1,2, \ldots, s ; m=0,1, \ldots, \bar{\ell}-1 ; j^{\prime}=1,2, \ldots, s^{\prime}}$ is a frame for $\ell_{N}^{2}(\mathbb{Z}) \otimes \ell_{M}^{2}(\mathbb{Z})$.

The thesis of the above proposition is true if and only if the $s \ell \times N$ matrix $\mathbf{R}_{\mathbf{a}, \mathbf{h}_{\mathbf{1}}}$ (defined in (24) for $\mathbb{G}_{j, n}^{1}$ ) has rank
$N$ and the $s^{\prime} \bar{\ell} \times M$ matrix $\mathbf{R}_{\mathbf{b}, \mathbf{h}_{2}}$ (defined in (24) for $\mathbb{G}_{j^{\prime}, m}^{2}$ ) has rank $M$. Notice that, necessarily, $s \geq r$ and $s^{\prime} \geq r^{\prime}$ and $s s^{\prime} \ell \bar{\ell} \geq N M=\operatorname{dim}\left(\ell_{N}^{2}(\mathbb{Z}) \otimes \ell_{M}^{2}(\mathbb{Z})\right)$.

5.1. The Sampling Result. In case that the sequence $\left\{\mathbb{G}_{j, n}^{1} \otimes \mathbb{G}_{j^{\prime}, m}^{2}\right\}_{n=0,1, \ldots, \ell-1 ; j=1,2, \ldots, s ; m=0,1, \ldots, \bar{\ell}-1 ; j^{\prime}=1,2, \ldots, s^{\prime}}$ is a frame for $\ell_{N}^{2}(\mathbb{Z}) \otimes \ell_{M}^{2}(\mathbb{Z})$, a family of appropriate dual frames is

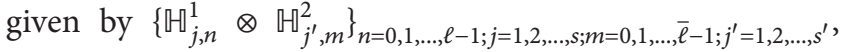

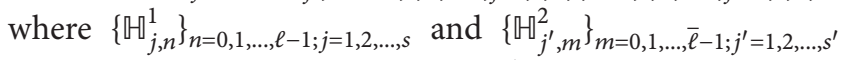
are appropriate dual frames of $\left\{\mathbb{G}_{j, n}^{1}\right\}_{n=0,1, \ldots, \ell-1 ; j=1,2, \ldots, s}$ and $\left\{\mathbb{G}_{j^{\prime}, m}^{2}\right\}_{m=0,1, \ldots, \bar{\ell}-1 ; j^{\prime}=1,2, \ldots, s^{\prime}}$, respectively. These dual frames are constructed as in Section 3.1 (see [3, Section 3.1] for the details).

Thus, for each $x \in \mathscr{A}_{a, b}$ there exists a unique $F \in \ell_{N}^{2}(\mathbb{Z}) \otimes$ $\ell_{M}^{2}(\mathbb{Z})$ such that $\mathscr{T}_{a, b}^{U V} F=x$. This $F$ can be expressed as the frame expansion

$$
F=\sum_{j=1}^{s} \sum_{j^{\prime}=1}^{s^{\prime}} \sum_{n=0}^{\ell-1} \sum_{m=0}^{\bar{\ell}-1} \mathscr{L}_{j j^{\prime}} x(r n, \bar{r} m)\left(\mathbb{U}_{j, n}^{1} \otimes \mathbb{E}_{j^{\prime}, m}^{2}\right)
$$

$$
\text { in } \ell_{N}^{2}(\mathbb{Z}) \otimes \ell_{M}^{2}(\mathbb{Z}) \text {. }
$$

Then, applying the isomorphism $\mathscr{T}_{a, b}^{U V}$ and (69) one gets

$$
\begin{aligned}
& x=\sum_{j=1}^{s} \sum_{j^{\prime}=1}^{s^{\prime}} \sum_{n=0}^{\ell-1} \sum_{m=0}^{\bar{\ell}-1} \mathscr{L}_{j j^{\prime}} x(r n, \bar{r} m) \mathscr{T}_{a, b}^{U V}\left(\mathbb{H}_{j, n}^{1} \otimes \mathbb{U}_{j^{\prime}, m}^{2}\right) \\
& =\sum_{j=1}^{s} \sum_{j^{\prime}=1}^{s^{\prime}} \sum_{n=0}^{\ell-1} \sum_{m=0}^{\bar{\ell}-1} \mathscr{L}_{j j^{\prime}} x(r n, \bar{r} m) U^{r n}\left(\mathscr{T}_{a, N}^{U} \mathbb{\boxplus}_{j, 0}^{1}\right) \otimes V^{\bar{r} m}\left(\mathscr{T}_{b, M}^{V} \mathbb{E}_{j^{\prime}, 0}^{2}\right) \\
& =\sum_{j=1}^{s} \sum_{j^{\prime}=1}^{s^{\prime}} \sum_{n=0}^{\ell-1} \sum_{m=0}^{\bar{\ell}-1} \mathscr{L}_{j j^{\prime}} x(r n, \bar{r} m)\left(U^{r n} \otimes V^{\bar{r} m}\right)\left(c_{j} \otimes d_{j^{\prime}}\right),
\end{aligned}
$$

where $c_{j}=\mathscr{T}_{a, N}^{U} \mathbb{E}_{j, 0}^{1} \in \mathscr{A}_{a}, j=1,2, \ldots, s$, and $d_{j^{\prime}}=$ $\mathscr{T}_{b, M}^{V} \mathbb{R}_{j^{\prime}, 0}^{2} \in \mathscr{A}_{b}, j^{\prime}=1,2, \ldots, s^{\prime}$. Next, we state the equivalent result to Theorem 6 .

Theorem 12. Let $h_{j j^{\prime}}:=h_{j 1} \otimes h_{j^{\prime} 2} \in \mathscr{H}_{1} \otimes \mathscr{H}_{2}, j=$ $1,2, \ldots, s, j^{\prime}=1,2, \ldots, s^{\prime}$, and let $\mathscr{L}_{j j^{\prime}}$ be the associated $U V$-system giving the samples of any $x \in \mathscr{A}_{a, b}$ as in (67), $j=1,2, \ldots, s, j^{\prime}=1,2, \ldots, s^{\prime}$. The following statements are equivalent:

(a) $\operatorname{rank} \mathbf{R}_{\mathbf{a}, \mathbf{h}_{1}}=N$ and $\operatorname{rank} \mathbf{R}_{\mathbf{b}, \mathbf{h}_{2}}=M$.

(b) There exist $S=s s^{\prime}$ elements $c_{j} \otimes d_{j^{\prime}}, j=$ $1,2, \ldots, s$ and $j^{\prime}=1,2, \ldots, s^{\prime}$, in the subspace $\mathscr{A}_{a, b}$ such that the sequence $\left\{\left(U^{r n} \otimes V^{\bar{r} m}\right)\left(c_{j} \otimes\right.\right.$

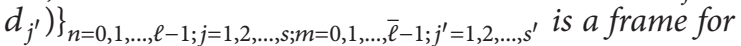
$\mathscr{A}_{a, b}$, and for any $x \in \mathscr{A}_{a, b}$ the expansion $x$

$$
=\sum_{j=1}^{s} \sum_{j^{\prime}=1}^{s^{\prime}} \sum_{n=0}^{\ell-1} \sum_{m=0}^{\bar{\ell}-1} \mathscr{L}_{j j^{\prime}} x(r n, \bar{r} m)\left(U^{r n} \otimes V^{\bar{r} m}\right)\left(c_{j} \otimes d_{j^{\prime}}\right)
$$

holds.

(c) There exists a frame

$\left\{C_{j, j^{\prime}, n, m}\right\}_{n=0,1, \ldots, \ell-1 ; j=1,2, \ldots, s ; m=0,1, \ldots, \bar{\ell}-1 ; j^{\prime}=1,2, \ldots, s^{\prime}} \quad$ for $\mathscr{A}_{a, b}$ such that, for each $x \in \mathscr{A}_{a, b}$, the expansion

$$
x=\sum_{j=1}^{s} \sum_{j^{\prime}=1}^{s^{\prime}} \sum_{n=0}^{\ell-1} \sum_{m=0}^{\bar{e}-1} \mathscr{L}_{j j^{\prime}} x(r n, \bar{r} m) C_{j, j^{\prime}, n, m}
$$

holds. 
As in the previous cases, whenever $s=r$ and $s^{\prime}=\bar{r}$ in the above theorem we are in the Riesz bases setting necessarily, and condition (a) says that both square matrices $\mathbf{R}_{\mathbf{a}, \mathbf{h}_{\mathbf{1}}}$ and $\mathbf{R}_{\mathbf{b}, \mathbf{h}_{\mathbf{2}}}$ are invertible.

A Representative Example. In $\mathscr{H}_{1}:=\ell_{N}^{2}(\mathbb{Z})$ consider the (circular) shift operator $U: x(p) \mapsto x(p-1)$ and $a:=$ $(1,0, \ldots, 0) \in \ell_{N}^{2}(\mathbb{Z})$ which obviously satisfies $U^{N} a=a$ and $\mathscr{A}_{a}=\ell_{N}^{2}(\mathbb{Z})$. Analogously, in $\mathscr{H}_{2}:=\ell_{M}^{2}(\mathbb{Z})$ consider the (circular) shift operator $V: y(q) \mapsto y(q-1)$, and $b:=(1,0, \ldots, 0) \in \ell_{M}^{2}(\mathbb{Z})$ which obviously satisfies $V^{M} b=b$ and $\mathscr{A}_{b}=\ell_{M}^{2}(\mathbb{Z})$.

Given the sequences $\mathbf{h}_{j 1} \in \ell_{N}^{2}(\mathbb{Z}), j=1,2, \ldots, s$, and $\mathbf{h}_{j 2} \in \ell_{M}^{2}(\mathbb{Z}), j^{\prime}=1,2, \ldots, s^{\prime}$, and fixing the sampling periods $r \mid N$ and $\bar{r} \mid M$ (recall that $\ell=N / r$ and $\bar{\ell}=M / \bar{r}$ ), for each $\mathbf{x}$ in $\ell_{N}^{2}(\mathbb{Z}) \otimes \ell_{M}^{2}(\mathbb{Z})=\ell_{N, M}^{2}\left(\mathbb{Z}^{2}\right)$, a double sequence in $\mathbb{Z}^{2}, N$-periodic in the first component, and $M$-periodic in the second one, we consider its samples defined by

$$
\begin{aligned}
\mathscr{L}_{j j^{\prime}} \mathbf{x}(r n, \bar{r} m) \\
:=\sum_{p=0}^{N-1} \sum_{q=0}^{M-1} \mathbf{x}(p, q) \overline{\mathbf{h}_{j 1}(p-r n)} \overline{\mathbf{h}_{j^{\prime} 2}(q-\bar{r} m)},
\end{aligned}
$$

where $n=0,1, \ldots, \ell-1, m=0,1, \ldots, \bar{\ell}-1, j=1,2, \ldots, s$, and $j^{\prime}=1,2, \ldots, s^{\prime}$.

Under the hypotheses in Theorem 12 there will be sequences $\mathbf{c}_{j} \in \ell_{N}^{2}(\mathbb{Z}), j=1,2, \ldots, s$, and $\mathbf{d}_{j^{\prime}} \in \ell_{M}^{2}(\mathbb{Z})$, $j^{\prime}=1,2, \ldots, s^{\prime}$, such that for any $\mathbf{x} \in \ell_{N, M}^{2}\left(\mathbb{Z}^{2}\right)$ the sampling expansion (78) reads as follows:

$$
\begin{aligned}
& \mathbf{x}(p, q) \\
& =\sum_{j=1}^{s} \sum_{j^{\prime}=1}^{s^{\prime}} \sum_{n=0}^{\ell-1} \sum_{m=0}^{\bar{\ell}-1} \mathscr{L}_{j j^{\prime}} \mathbf{x}(r n, \bar{r} m) \mathbf{c}_{j}(p-r n) \mathbf{d}_{j^{\prime}}(q-\bar{r} m),
\end{aligned}
$$

where $0 \leq p \leq N-1$ and $0 \leq q \leq M-1$.

\section{Discussion and Conclusions}

A sampling theory for tensor products of unitary invariant subspaces that allows merging the cases of finitely/infinitely generated unitary invariant subspaces formerly studied in the mathematical literature is derived. The involved samples are identified as frame coefficients in suitable tensor product spaces; thus it also allows introducing the several variables case in the formalism.

Alternatively, the theory developed here can also be considered as a theory of invariant subspaces in the tensor product of the corresponding Hilbert space with respect to the canonical unitary tensor product representation of the product group $\mathbb{Z} \otimes \mathbb{Z}_{p}$ defined from the corresponding factors (in the case of infinite-finite generators).

In this sense, the results exhibited here point again in the direction recently stated in the paper [19] and natural generalizations of them, like sampling theorems for tensor products of invariant subspaces with respect to unitary representations of finite or infinite groups on each factor, can be addressed using similar ideas.

Reduction techniques corresponding to the decomposition of tensor products of unitary representations into their irreducible components with respect to proper subgroups of the product group could also be used to simplify the construction of samples, that is, to find adapted frames. This, as well as other applications beyond classical telecommunications all involving sampling of states of quantum systems, will be the subject of further research.

\section{Competing Interests}

The authors declare that they have no competing interests.

\section{Acknowledgments}

This work has been supported by the Grant MTM201454692-P from the Spanish Ministerio de Economía y Competitividad (MINECO).

\section{References}

[1] H. R. Fernández-Morales, A. G. García, M. A. HernándezMedina, and M. J. Muñoz-Bouzo, "On some sampling-related frames in $U$-invariant spaces," Abstract and Applied Analysis, vol. 2013, Article ID 761620, 14 pages, 2013.

[2] H. R. Fernández-Morales, A. G. García, M. A. HernándezMedina, and M. J. Muñoz-Bouzo, "Generalized sampling: from shift-invariant to $U$-invariant spaces," Analysis and Applications, vol. 13, no. 3, pp. 303-329, 2015.

[3] A. G. García and M. Muñoz-Bouzo, "Sampling-related frames in finite U-invariant subspaces," Applied and Computational Harmonic Analysis, vol. 39, no. 1, pp. 173-184, 2015.

[4] T. Michaeli, V. Pohl, and Y. C. Eldar, "U-invariant sampling: extrapolation and causal interpolation from generalized samples," IEEE Transactions on Signal Processing, vol. 59, no. 5, pp. 2085-2100, 2011.

[5] V. Pohl and H. Boche, "U-invariant sampling and reconstruction in atomic spaces with multiple generators," IEEE Transactions on Signal Processing, vol. 60, no. 7, pp. 3506-3519, 2012.

[6] A. Aldroubi, "Non-uniform weighted average sampling and reconstruction in shift-invariant and wavelet spaces," Applied and Computational Harmonic Analysis, vol. 13, no. 2, pp. 151161,2002

[7] A. Aldroubi and K. Gröchenig, "Nonuniform sampling and reconstruction in shift-invariant spaces," SIAM Review, vol. 43, no. 4, pp. 585-620, 2001.

[8] O. Christensen, An Introduction to Frames and Riesz Bases, Applied and Numerical Harmonic Analysis, Birkhäuser, Boston, Mass, USA, 2003.

[9] H. R. Fernández-Morales, A. G. García, and G. Pérez-Villalón, "Generalized sampling in $L^{2}\left(\mathbb{R}^{d}\right)$ shift-invariant subspaces with multiple stable generators," in Multiscale Signal Analysis and Modeling, Lecture Notes in Electrical Engineering, pp. 51-80, Springer, New York, NY, USA, 2012.

[10] A. G. García and G. Pérez-Villalón, "Dual frames in $L^{2}(0,1)$ connected with generalized sampling in shift-invariant spaces," 
Applied and Computational Harmonic Analysis, vol. 20, no. 3, pp. 422-433, 2006.

[11] A. G. García, M. A. Hernández-Medina, and G. Pérez-Villalón, "Generalized sampling in shift-invariant spaces with multiple stable generators," Journal of Mathematical Analysis and Applications, vol. 337, no. 1, pp. 69-84, 2008.

[12] S. Kang and K. H. Kwon, "Generalized average sampling in shift invariant spaces," Journal of Mathematical Analysis and Applications, vol. 377, no. 1, pp. 70-78, 2011.

[13] C. V. M. van der Mee, M. Z. Nashed, and S. Seatzu, "Sampling expansions and interpolation in unitarily translation invariant reproducing kernel Hilbert spaces," Advances in Computational Mathematics, vol. 19, no. 4, pp. 355-372, 2003.

[14] Q. Sun, "Local reconstruction for sampling in shift-invariant spaces," Advances in Computational Mathematics, vol. 32, no. 3, pp. 335-352, 2010.

[15] W. Sun and X. Zhou, "Average sampling in spline subspaces," Applied Mathematics Letters, vol. 15, no. 2, pp. 233-237, 2002.

[16] W. Sun and X. Zhou, "Average sampling in shift invariant subspaces with symmetric averaging functions," Journal of Mathematical Analysis and Applications, vol. 287, no. 1, pp. 279295, 2003.

[17] X. Zhou and W. Sun, "On the sampling theorem for wavelet subspaces," The Journal of Fourier Analysis and Applications, vol. 5, no. 4, pp. 347-354, 1999.

[18] H. R. Fernández-Morales, A. G. García, M. J. Muñoz-Bouzo, and A. Ortega, "Finite sampling in multiple generated Uinvariant subspaces," IEEE Transactions on Information Theory, vol. 62, no. 4, pp. 2203-2212, 2016.

[19] A. G. García, A. Miguel, and A. Ibort, "Towards a quantum sampling theory: the case of finite groups," https://arxiv.org/abs/1510 $.08134 \mathrm{vl}$.

[20] F. Natterer and F. Wübbeling, Mathematical Methods in Image Reconstruction, SIAM, Philadelphia, Pa, USA, 2001.

[21] H. Stark, "Polar, spiral, and generalized sampling and interpolation," in Advanced topics in Shannon Sampling and Interpolation Theory, R. J. Marks II, Ed., pp. 185-207, Springer, New York, NY, USA, 1993.

[22] R. J. Duffin and A. C. Schaeffer, "A class of nonharmonic Fourier series," Transactions of the American Mathematical Society, vol. 72, pp. 341-366, 1952.

[23] I. Daubechies, A. Grossmann, and Y. Meyer, "Painless nonorthogonal expansions," Journal of Mathematical Physics, vol. 27, no. 5, pp. 1271-1283, 1986.

[24] P. G. Casazza and G. Kutyniok, Eds., Finite Frames: Theory and Applications, Birkhäuser, Boston, Mass, USA, 2014.

[25] G. B. Folland, A Course in Abstract Harmonic Analysis, CRC Press, Boca Raton, Fla, USA, 1995.

[26] A. Bourouihiya, "The tensor product of frames," Sampling Theory in Signal and Image Processing, vol. 7, no. 1, pp. 65-76, 2008.

[27] A. Khosravi and M. S. Asgari, "Frames and bases in tensor product of Hilbert spaces," International Mathematical Journal, vol. 4, no. 6, pp. 527-537, 2003.

[28] C. S. Kubrusly, "A concise introduction to tensor product," Far East Journal of Mathematical Sciences, vol. 22, no. 2, pp. 137-174, 2006.

[29] A. N. Kolmogorov, "Stationary sequences in Hilbert space," Moscow University Mathematics Bulletin, vol. 2, pp. 1-40, 1941.
[30] R. Penrose, "A generalized inverse for matrices," Mathematical Proceedings of the Cambridge Philosophical Society, vol. 51, no. 3, pp. 406-413, 1955.

[31] A. G. García, "Sampling theory and reproducing kernel Hilbert spaces," in Operator Theory, D. Alpay, Ed., pp. 87-110, Springer, Basel, Switzerland, 2015. 


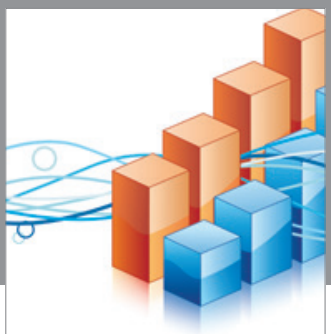

Advances in

Operations Research

vatem alat4

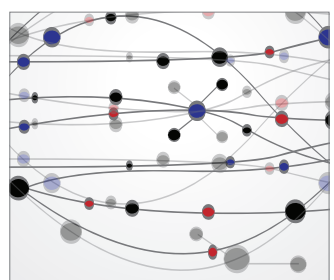

\section{The Scientific} World Journal
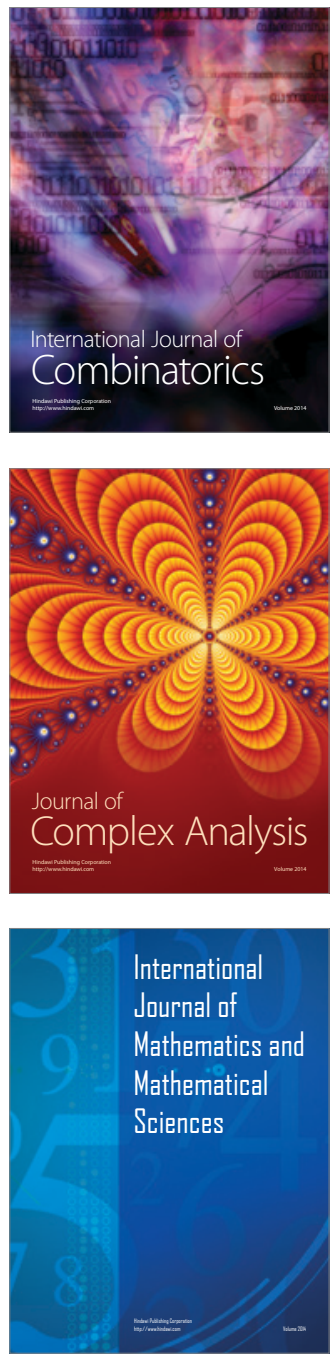
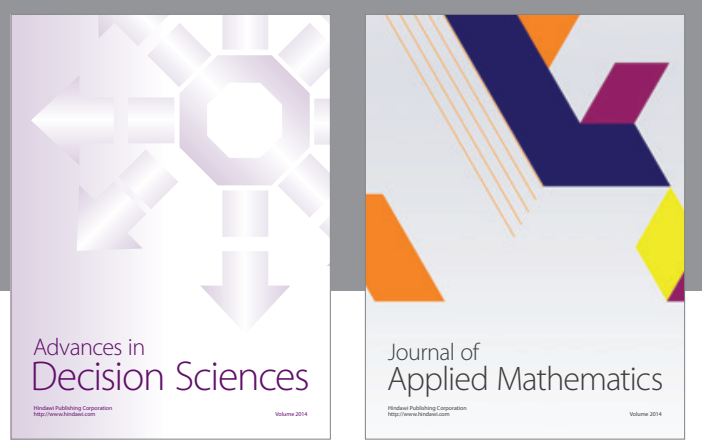

Algebra

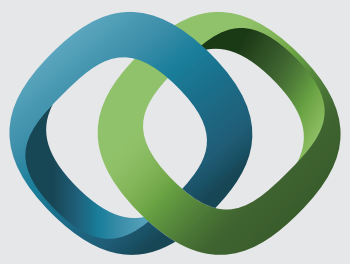

\section{Hindawi}

Submit your manuscripts at

http://www.hindawi.com
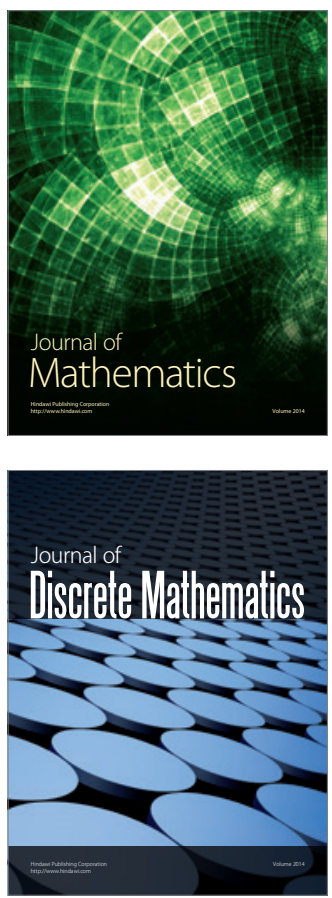

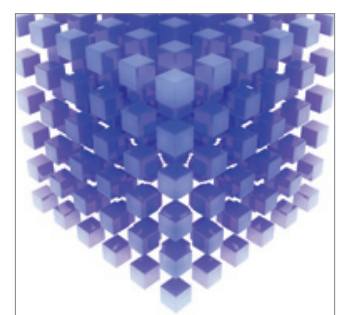

Mathematical Problems in Engineering
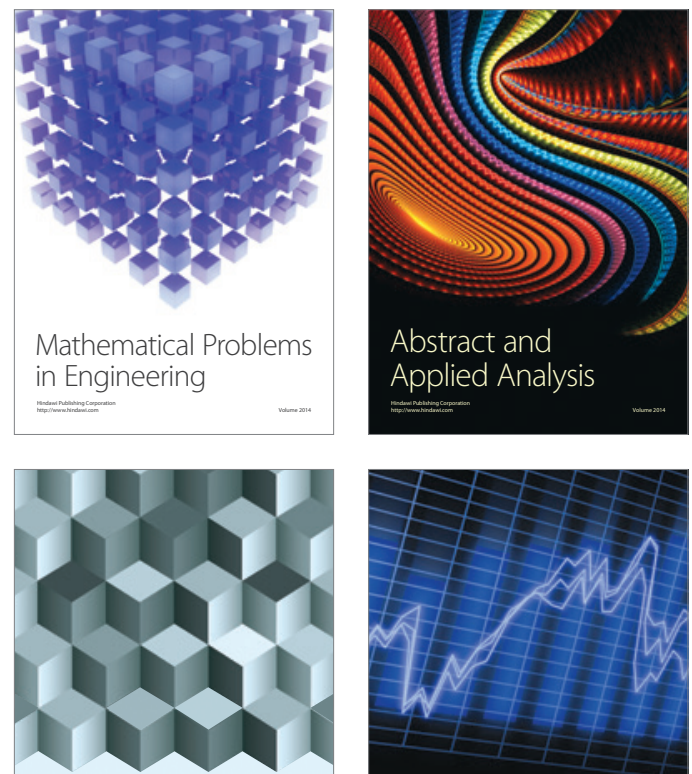

Journal of

Function Spaces

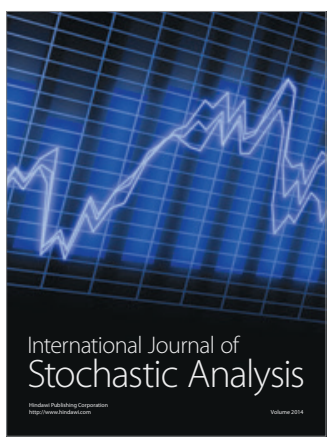

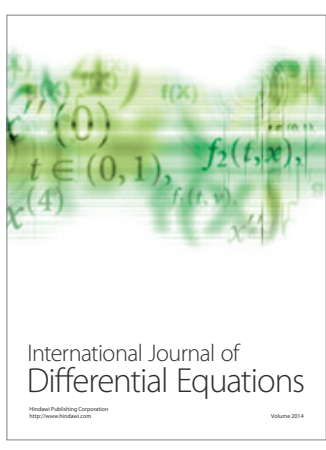
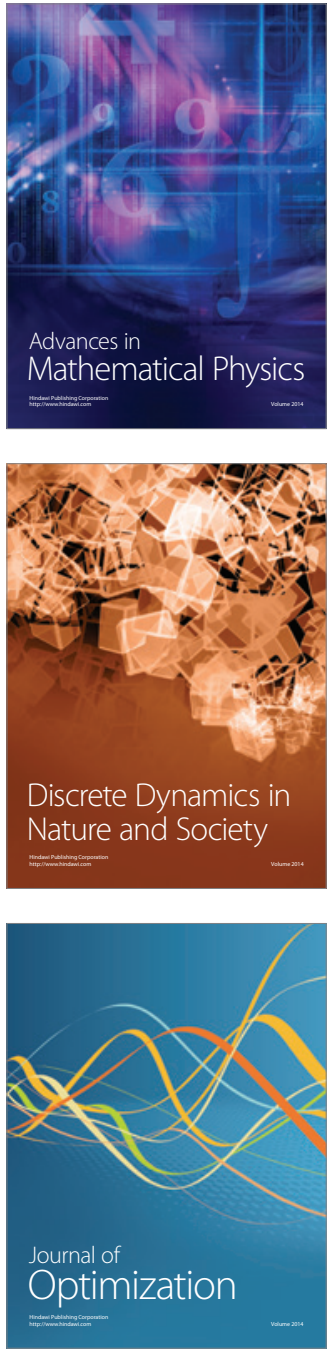